\title{
The direct observation of point defects in irradiated or quenched metals by quantitative field ion microscopy $\dagger$
}

\author{
DAVID N SEIDMAN $\ddagger$ \\ Department of Materials Science and Engineering, Cornell University, Ithaca, New York \\ 14850 , USA
}

MS received 21 September 1972

\begin{abstract}
The progress made at Cornell University over the past few years in applying the field ion microscope (FIM) technique to the study of point defects in irradiated or quenched metals is reviewed. The techniques involve essentially the atom by atom dissection of specimens by pulse field evaporation and the corresponding recording on film of the extensive number of FM images produced at each stage of the process. Methods have been developed which allow the recording and the analysis of as many as approximately $5 \times 10^{5}$ frames of $35 \mathrm{~mm}$ cine film for a single investigation. The application of these techniques to the following problems were discussed: (i) the mobility of self interstitial atoms (SIA) in substage $\mathrm{I}_{\mathrm{E}}$ of irradiated tungsten and platinum, (ii) the geometric configuration of the SIA, (iii) the point defect structure of depleted zones in tungsten and (iv) the properties of monovacancies and divacancies in quenched platinum.
\end{abstract}

\section{Introduction}

The field ion microscope (FIM) with its excellent atomic resolution is ideally suited for the study of point defects (see Hren and Ranganathan 1968, Müller and Tsong 1969 and Bowkett and Smith 1970 for texts on the FIM technique). FIM studies are capable of yielding direct information pertaining to the atomic mechanisms responsible for the recovery of irradiated or quenched metals. The most difficult aspect of these studies is the retrieval of the information which is contained within the small FLM tips that are studied. To overcome this problem we have automated the atom by atom dissection of specimens by the pulsed field technique and the simultaneous recording on film of the extensive number of FIM images produced at each stage of the process. Methods have also been developed which allow the recording and analysis of as many as approximately $5 \times 10^{5}$ frames of cine film for a single investigation. In view of the well known perils of interpreting FIM images, a strong effort was made to examine a large number of control specimens prior to both the irradiation and quenching experiments. The control experiments played a crucial role in that they allowed us to interpret, with confidence, the results presented in this review article.

$\therefore$ Research supported by the United States Atomic Energy Commission. Additional support was received from the Advanced Research Projects Agency through the use of the technical facilities of the Materials Science Center at Cornell University.

$\ddagger$ John Simon Guggenheim Memorial Foundation Fellow 1972-73. 
The application of these techniques to the following problems are discussed in the present paper: (i) the mobility of self interstitial atoms (SIA) in substage $I_{E}$ of irradiated tungsten and platinum; (ii) the geometric configuration of SIA's: (iii) the point defect structure of depleted zones in tungsten and (iv) the properties of monovacancies and divacancies in quenched platinum. In addition to these problems the following studies are in progress in our laboratory: (i) the mobility of the SIA in stage I and III of gold, (ii) the mobility of the SIA in stage III of platinum, tungsten and nickel, (iii) the measurement of binding energies of the SIA to impurity atoms and (iv) monovacancies and divacancies in quenched tungsten (Balluffi, research in progress).

\section{A brief review of point defect mechanisms and problems associated with the recovery of irradiated metals}

\subsection{Point defect mechanisms for recovery of irradiated face centred cubic metals}

The stage I recovery spectra of electron, thermal neutron, deuteron or fast neutron irradiated fcc metals at low doses can exhibit as many as five substages $\left(I_{A}, I_{B}, I_{C}, I_{D}\right.$ and $I_{E}$ ) (eg see Schilling et al 1970). The generally accepted mechanism for the substages $I_{A}$ to $I_{C}$ is the recombination of a monovacancy and an SIA which are members of a close Frenkel pair. Each of the first three substages corresponds to a recombination of Frenkel pairs with a progressively higher energy barrier between the monovacancy and the SIA. The mechanisms responsible for substages $I_{D}$ and $I_{E}$ are still subject to considerable controversy (Corbett 1970, Koehler 1970, Seeger 1970, Frank and Seeger 1969, Schüle et al 1971, Wollenberger 1971). One interpretation for $I_{D}$ and $I_{E}$ is based on the Corbett et al (1959a, 1959b) model which attributes recovery in these two substages to a SIA which migrates in three dimensions and undergoes correlated recombination with a monovacancy in $I_{D}$ and uncorrelated recombination with a monovacancy in $I_{E}$. This model assumes that the SIA which migrates in stage $I$ is the only stable one produced by the irradiation processes and is known as the 'one interstitial' model. The alternative explanation is referred to as the 'two interstitial' model. One version of this model involves the production of a metastable SIA (the crowdion) which migrates in one dimension in $I_{D}$ and $\mathrm{I}_{E}$, and can also convert to a stable form (a split SIA) which subsequently undergoes three dimensional long range migration in stage III. A recent modification of this model (Frank 1971) asserts that off line crowdions $\nmid$ are responsible for $I_{E}$ while on line crowdions are responsible for $I_{D}$. Another variation (Simpson and Sosin 1970a, 1970b) of the 'two interstitial' model states that a metastable three dimensional sIA migrates in stage $I\left(I_{D}\right.$ is correlated and $\mathrm{I}_{\mathrm{E}}$ is uncorrelated migration) and that this SIA also undergoes thermal conversion to a second interstitial configuration which subsequently migrates in three dimensions in stage III. A common feature of all three models is the necessity of a SIA which can undergo uncorrelated long range migration in $I_{E}$ and it is to this recovery problem that we have applied the FIM technique, for the metals platinum (Pétroff and Seidman 1971, 1972a) and gold (Averback and Seidman, research in progress).

Platinum was chosen as a prototype foc metal because of its amenability to the FIM technique and our ability to prepare ultra high purity single crystals of this metal. Gold is a difficult metal to examine by the FIM technique (Ast and Seidman 1968, 1970, 1971,

+ An off line crowdion is one that is refocussed into other crowdion directions after it has propagated away from its original site along a particular crowdion direction. 
Ast 1972 and Averback and Seidman, unpublished) but the macroscopic stage I recovery behaviour of gold is anomalous when compared to the other foc metals (eg see Schilling et al 1970, Venables 1970, Venables and Thomas 1970, Seeger 1970) and the question of long migration in stage $I$ of gold is still a very controversial topic.

\subsection{Point defect mechanisms for recovery of irradiated body centred cubic metals}

The stage I recovery spectra of irradiated bcc metals tend to be more complex than the recovery spectra of foc metals and the reproducibility of a given spectrum for a particular metal in different laboratories has been poor (eg compare the electron irradiation experiments on tungsten of Neely et al 1968, Roberts 1968a, 1968b, and DiCarlo et al 1969). The interpretation of stage I recovery behaviour in bcc metals has been influenced by the research on fcc metals and it is common to associate uncorrelated long range migration of an SIA with a peak which is referred to as substage $\mathrm{I}_{\mathrm{E}}$ in analogy with the fcc metals (eg see Schultz 1968/69 and Nihoul 1970).

We chose tungsten as a prototype bcc metal because it is easily studied in the FIM and because it can be purified to a level of about $(0 \cdot 1-1) \times 10^{-6}$ at fr impurities. In addition there existed a substantial literature on the macroscopic stage I recovery behaviour of irradiated tungsten.

\section{The direct observation of uncorrelated long range migration in substage $I_{E}$ of irradiated fcc and bec metals}

\subsection{Principles of the experiments employed to detect uncorrelated long range migration in substage $I_{E}$}

The three experimental steps employed in the detection of uncorrelated long range migration in $\mathrm{I}_{\mathrm{E}}$ for both fcc and bcc metals are illustrated schematically in figures $1(a)$ to 1(c). These steps and their physical significance were as follows:

(i) A specimen was irradiated in situ under ultra high vacuum conditions in the absence of an electric field $(E)$ and at a temperature $\left(T_{0}\right)$ which was below the onset of any recovery as determined from the published isochronal recovery spectra. The irradiating particle was a magnetically analysed $20-40 \mathrm{keV}$ singly charged metal ion $\left(\mathrm{M}^{+}\right)$which had the same atomic mass as the specimen being irradiated (eg $\mathrm{W}^{+}$ion for a tungsten specimen). The use of the $\mathbf{M}^{+}$ion to irradiate $\mathrm{M}$ was essential, since this decreased strongly the probability of introducing impurities as a result of the bombardment. The total dose was between $1 \times 10^{10}$ and $1 \times 10^{13}$ ion $\mathrm{cm}^{-2}$. Details regarding the cryogenic techniques, the irradiation facility employed and temperature measurement can be found in Seidman et al (1969), Scanlan et al (1971a), and Seidman and Scanlan (1971).

(ii) The initial state of damage produced at $T_{0}$ was determined via the atom by atom pulsed field evaporation technique. The damage pattern consisted of depleted zones within about $100 \AA$ of the irradiated surface and a distribution of immobile sIA in the bulk of the specimen. The sIA most likely originated at the depleted zones and travelled relatively long distances as the result of focused collision replacement sequences (eg see Seeger 1962, Beeler 1966 and Venables 1970 for theory and Venables 1970 and Beavan et al 1971 for experimental evidence). The object here was to determine first if immobile SIA could be found in the bulk of a specimen at $T_{0}$ prior to the isochronal warming experiment. Information regarding the pulse field evaporation technique and the 


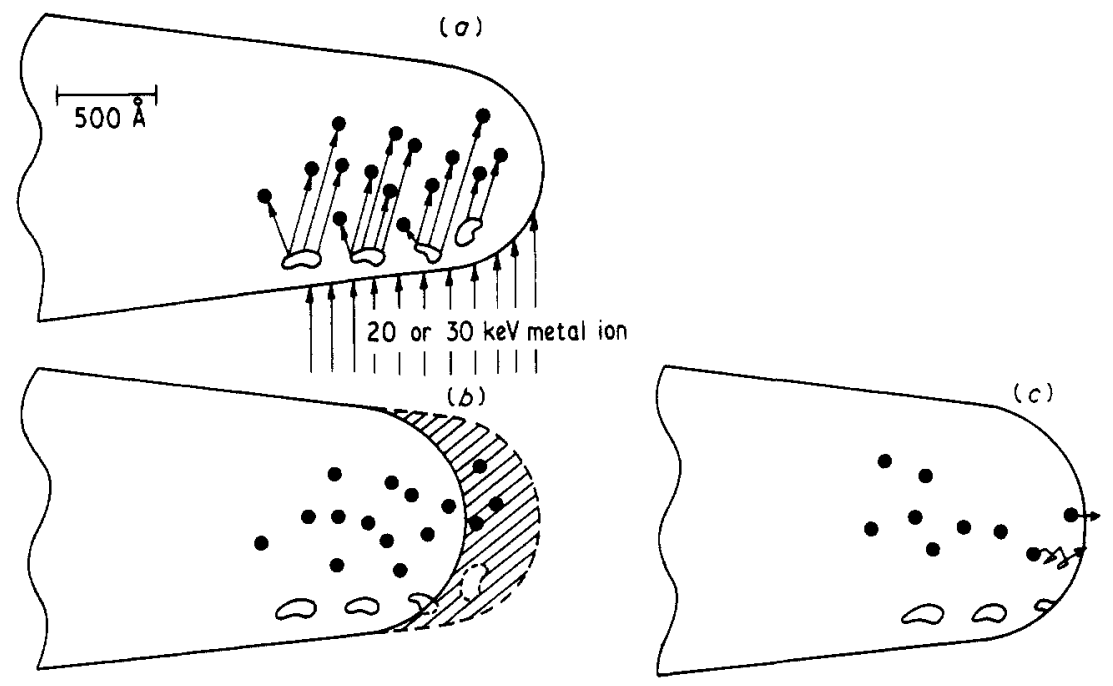

Figure 1. A schematic diagram of the field ion microscopic experiments performed to detect long range uncorrelated migration of SIA in substage $\mathbf{I}_{E}$ of an irradiated metal (see $\$ 3.2$ ). (a) shows in situ irradiation of the specimen at $T_{0}$. In $(b)$ the shaded area denotes the material examined during the pulse field evaporation experiment. (c) shows self interstitial atoms crossing the free surface during a continuous anneal from $T_{0}$.

semiautomated data recording and analysis system has been reported by Robertson and Seidman (1968) and Scanlan et al (1969).

(iii) After the completion of the pulse field evaporation experiments shown in figure 1 (b) the same specimen was warmed isochronally from $T_{0}$ through stage $I$ and the specimen's surface was photographed continuously at a rate of one frame per second. During the isochronal warming experiment the value of $E$ was maintained at the best image field (BIF) which was chatactistic of $T_{0}$. The purpose of this experiment was to determine the temperature range in stage $I$ in which the SIA migrated out of the bulk to the free surface; that is, to observe directly the long range migration of the SIA. During the isochronal warming experiment all the atoms on the surface were stable and all the SIA were detected as a result of their migrating to the surface which was the dominant sink. By comparison, in the dissection experiments (figure $1(b)$ ) all the SIA were detected as a result of the atom by atom examination of successive planes of the lattice. A discussion of the contrast effects produced by SIA detected by the dissection techniques is given in $\$ 6$.

In comparing an FIM isochronal spectrum to an isochronal recovery spectrum determined by electrical resistivity measurements it is emphasized that the FIM experiments can only detect SIA which undergo uncorrelated long range migration (substage $\mathrm{I}_{\mathrm{E}}$ ); that is, SIA which cross the specimen's surface during a prescribed isochronal procedure. In the case of heavy metal ion damage the substages $\mathrm{I}_{\mathrm{A}}$ to $\mathrm{I}_{\mathrm{C}}$ involve the recombination of Frenkel pairs within and around a depleted zone. These recombination events can be monitored by electrical resistivity measurement, but are not detectable by the FIM isochronal warming experiment. Any correlated recombination involving long range migration would also not be detectable via the FIM isochronal heating experiment, since this mechanism involves an SIA returning to its monovacancy in or near a depleted zone. Hence, the recovery spectra obtained are related to the substage $I_{E}$ peaks found by resistivity measurements and not the entire stage I recovery spectrum. 


\subsection{Uncorrelated long range migration in substage $I_{E}$ of tungsten}

3.2.1. Scanlan, Styris and Seidman. The stage I $\dagger$ experiments on tungsten (Scanlan et al 1971a, 1971b, Wilson and Seidman, unpublished) consisted of the irradiation of zone refined tungsten specimens with resistivity ratios $\mathscr{R}\left(\mathscr{R}=R_{293 \mathrm{~K}} / R_{4 \cdot 2 \mathrm{~K}}\right)$ of $(4$ to 5$) \times 10^{4}$ $\left(\leqslant 1.5 \times 10^{-6}\right.$ at $\mathrm{fr}$ impurities) at temperatures between 8 and $18 \mathrm{~K}$. The examination of the interior of these specimens at $T_{0}$ by the pulse field evaporation technique revealed the existence of immobile SIA distributed throughout a volume that extended about $200 \AA$ below the specimens' surfaces. A composite plot of the depth distribution of these SIA is shown in figure 2 for an average irradiation temperature $(\bar{T})$ of $15 \mathrm{~K}$. Direct observation

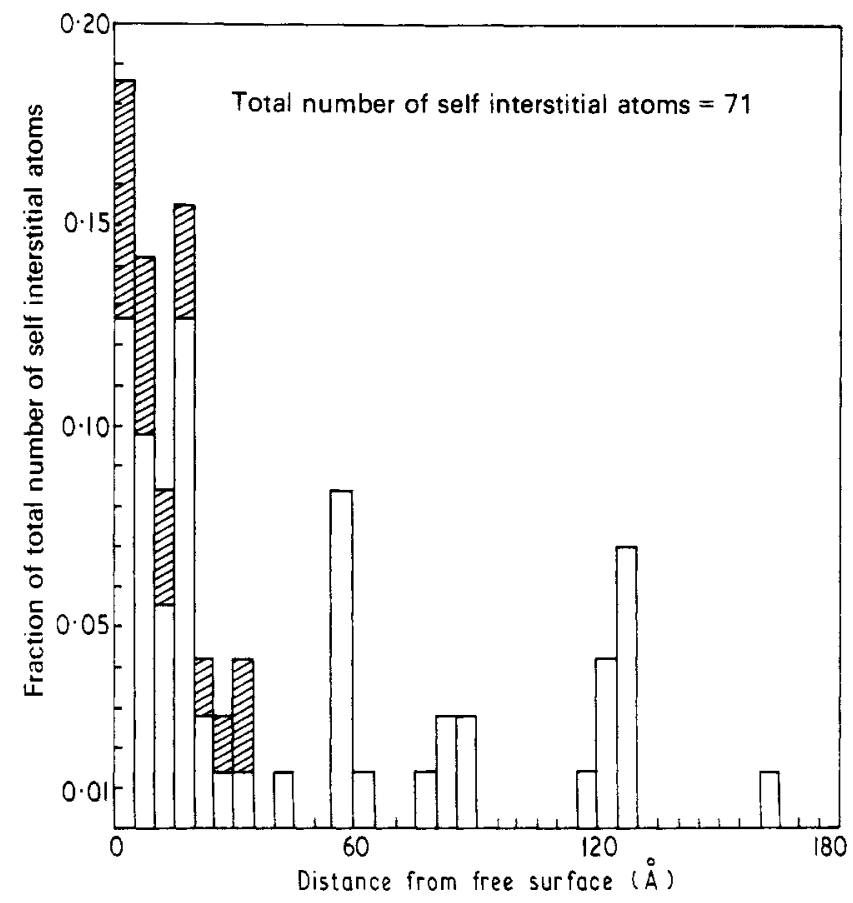

Figure 2. The depth distribution of SIA obtained from the field evaporation experiments on tungsten specimens irradiated at $\bar{T}_{0}=15 \mathrm{~K}$. The field evaporation was performed prior to the isochronal warming experiments. The fraction of the total number of defects found in each $5 \AA$ increment of material removed from the specimen was plotted as a function of the distance (in $\AA$ ) from the surface of the specimen. The quantity $\bar{T}_{0}$ is the average irradiation temperature. The shaded area shows self interstitial atoms detected near depleted zones.

of the unperturbed surfaces of the FIM specimens during the subsequent isochronal warming experiments in the absence of field evaporation demonstrated that SIA diffused to the specimens' surfaces and emerged there after undergoing uncorrelated long range migration. Figure $3(a)$ is a composite histogram of the fraction of the total number defects against the $5 \mathrm{~K}$ temperature in which the defects emerged at the surface for 11 specimens which were irradiated at $\bar{T}_{0}=15 \mathrm{~K}$ to doses that varied between $5 \times 10^{11}$ to $1 \times 10^{13} \mathrm{~W}^{+}$ion $\mathrm{cm}^{-2}$. The term defects is employed in this section as opposed to SIA, 
because clusters of bright spots were also observed to arrive at the surface during the isochronal warming experiments. Some of these clusters may have been disia or even trisiA. The approximate envelope of the histogram of figure $3(a)$ is shown in figure $3(b)$ and it is seen that the data exhibited a broad maximum centred at $\sim 38 \mathrm{~K}$ followed by a long tail which decayed to zero by $120 \mathrm{~K}$. The superimposed calculated peak shape exhibited in figure $3(b)$ is discussed in $\$ 4$.

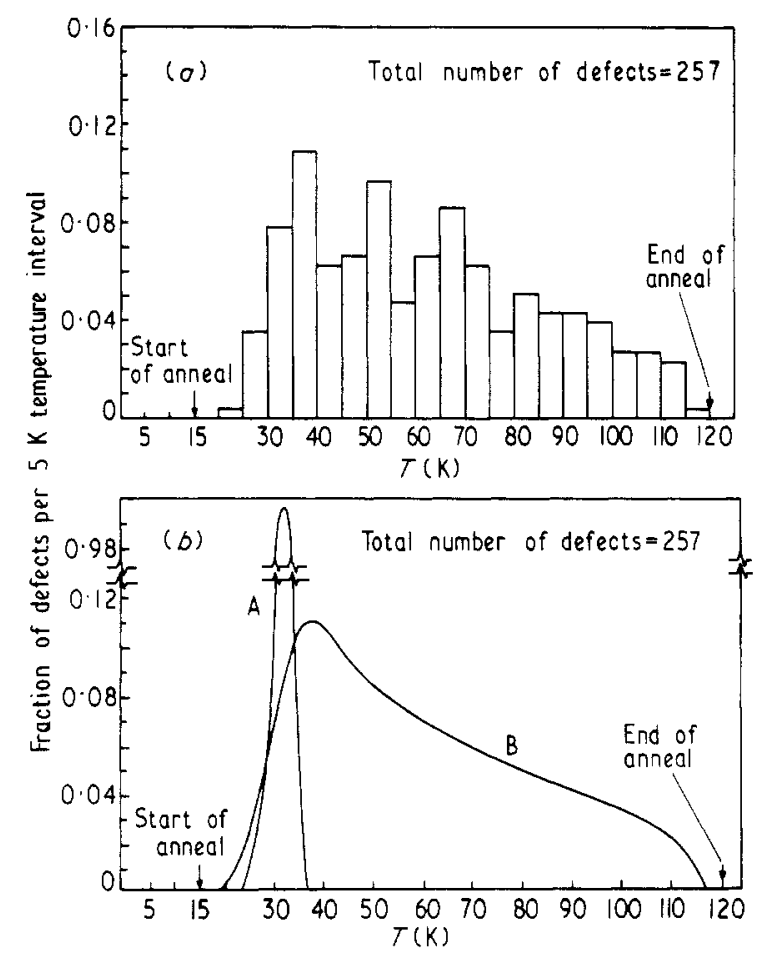

Figure 3. (a) The composite spectrum for the isochronal recovery of 11 tungsten specimens which were irradiated at $\sim 15 \mathrm{~K}$ with $20 \mathrm{keV} \mathrm{W}^{+}$ions and then annealed at a linear rate of $2 \mathrm{~K} \mathrm{~min}^{-1}$ to $120 \mathrm{~K}$. (b) Curves A and B are, respectively, a plot of the calculated peak shape $\left(\Gamma=10^{16}\right)$ superimposed on the envelope of the experimental data shown in $(a)$.

Examples of the appearance of SIA in tungsten emerging at or in the surface during the course of an isochronal warming experiment are shown in figure 4 (plate). The arrows in the figure point to either the positions where the sIA have almost emerged, or to the SIA which have emerged. The time interval between the two frames in all three sets of micrographs $((a),(b)$, and $(c))$ is $1 \mathrm{~s}$, which corresponds to a temperature interval of $0.03 \mathrm{~K}$. The simple single bright spot type of contrast (figures $4(a)$ and $4(b))$ was the most common contrast effect observed and was exhibited by $\sim 87 \%$ of the 257 defects observed. An example of a defect consisting of a triple extra bright spot is shown in figure $4(\mathrm{c})$ (only $\sim 1 \%$ of the 257 defects detected exhibited this type of contrast effect). In the isochronal warming experiments the contrast effects exhibited by the SIA were such that each SIA went from 'no contrast' to 'full contrast' within one frame of film. This effect was different from the contrast effects exhibited by SIA when they were detected by the pulse field evaporation technique (see $\$ 6$ ). 

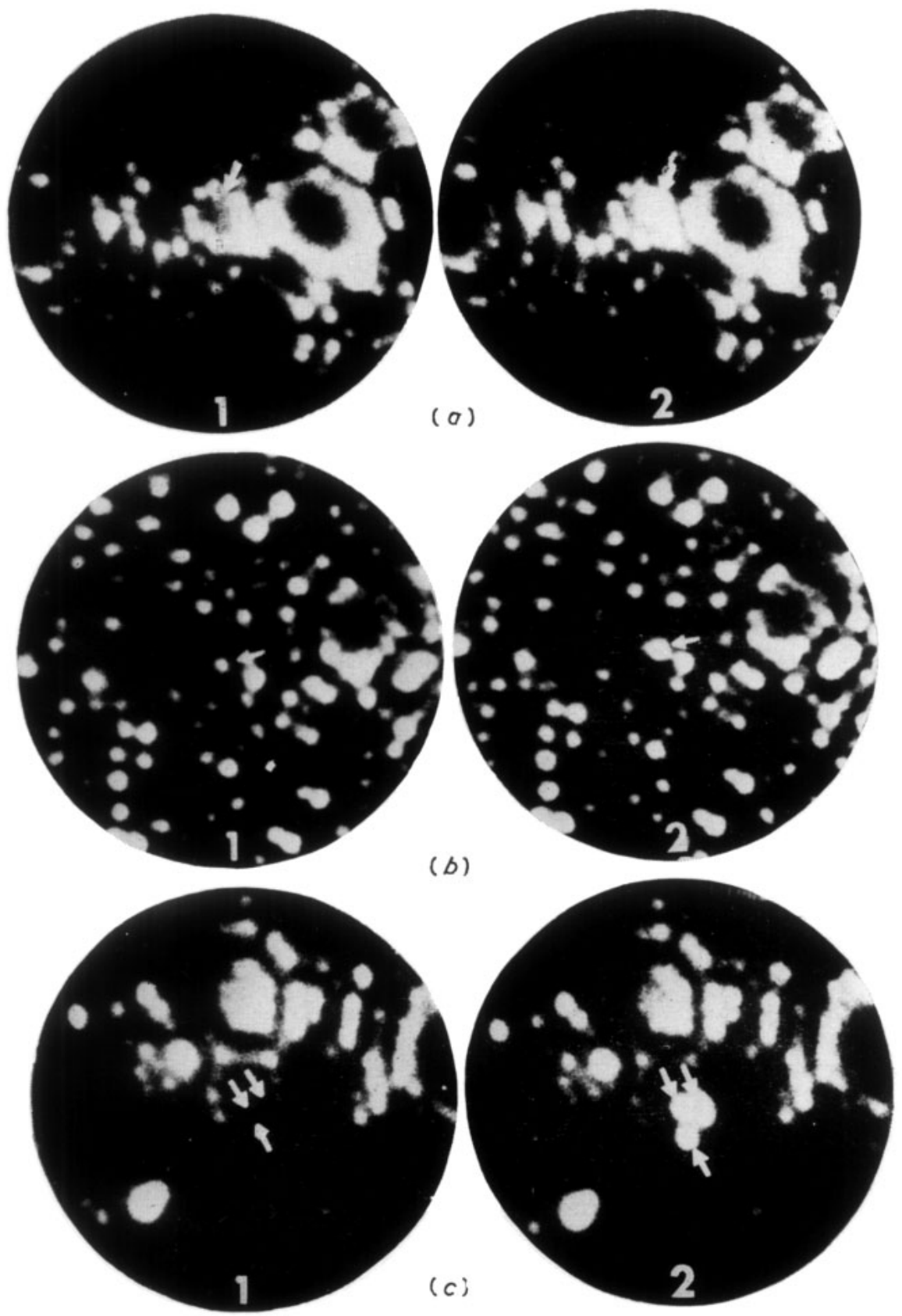

Figure 4. Three different examples of the emergence of SIA at the surface of a tungsten specimen during the course of an isochronal warming experiment between 15 and $120 \mathrm{~K}$. In the three cases shown in $(a),(b)$ and $(c)$ the temperature interval between micrograph 1 and 2 of each pair was $0.03 \mathrm{~K}$ and the time interval was $1 \mathrm{~s}$. The specimen was maintained at the BIF characteristic of $15 \mathrm{~K}$ during the entire warming experiment. 

Upon the completion of the isochronal warming experiments in stage I the specimens were warmed to $\sim 298 \mathrm{~K}$ (middle of stage II) and then recooled to $18 \mathrm{~K}$ for a post anneal field evaporation examination of the specimen bulk. These experiments showed that the bulk concentration of SIA decreased as a result of warming through stage I to the middle of stage II by at least a factor of 10 . Thus, two different types of SIA did not exist simultaneously in stage $I$.

3.2.2. Sinha and Müller. Sinha and Müller's (1964) pioneering FIM experiments consisted of the irradiation of tungsten with $20 \mathrm{keV}$ neutral helium atoms at $21 \mathrm{~K}$ in the presence of the BIF characteristic of this temperature. The authors state that 'stress enhanced mobility of interstitials is detectable at $21 \mathrm{~K}$ and rapid thermal diffusion from the depth occurs at $90 \mathrm{~K}$ '. There are two experimental problems with the Sinha-Müller experiments, which make it doubtful that they observed pure uncorrelated long range migration of SIA.

(i) The use of commercial tungsten wire (Thermonic Products Company NS wire) for the experiment. The value of $\mathscr{H}$ for this type of tungsten was probably less than 20 . Thus, the impurity level in their specimens was at least 2000 times greater than the impurity content in the specimens employed by Scanlan et al (1971a).

(ii) The use of helium atoms as the bombarding species is another source of impurities in the Sinha-Müller experiments. It is likely that the helium atoms became trapped (eg see Erents and Carter 1966, Erents et al 1967 and Kornelsen 1972) in the specimen at $21 \mathrm{~K}$ after they had expended all their energy in producing displaced lattice atoms.

The high impurity concentrations in the Sinha and Müller specimens made it very probable that the majority of the SIA became trapped at impurities before they ever reached the specimen's surface. The recovery peak which they observed at $90 \mathrm{~K}$ was most likely caused by the release of SIA from impurity traps, so that what they probably detected was the impurity delayed diffusion of SIA (Lie and Seidman, unpublished; see $\$ 5$ ) and not pure uncorrelated long range migration.

\subsection{Uncorrelated long range migration in substage $I_{E}$ of platinum}

The stage I experiments on platinum (Pétroff and Seidman 1971, 1972a) involved the in situ irradiation of high purity $\left((2-6) \times 10^{-5}\right.$ and $(1-3) \times 10^{-6}$ at $\mathrm{fr}$ impurity level) specimens with 20 or $30 \mathrm{keV} \mathrm{Pt}{ }^{+}$ions under ultrahigh vacuum conditions at $T_{0}=10$ or $11 \mathrm{~K}$. In a preanneal pulse field evaporation experiment + at $10 \mathrm{~K}$ on an irradiated specimen, a total of eight SIA were detected in the first $120 \AA$ of material examined (SIA concentration approximately $2 \times 10^{-5}$ at $\mathrm{fr}$ ). Hence, immobile SIA existed in the bulk of a specimen prior to warming it isochronally through stage I\$.

For the isochronal warming experiments a total of seven different specimens were irradiated at $T_{0}$ and then warmed from $T_{0}$ to $76 \mathrm{~K}$ in steps of $2 \mathrm{~K}$ and held at each tip temperature $\left(T_{\mathrm{T}}\right)$ for $6 \mathrm{~min}$. This corresponded to an average linear warming rate $\alpha$ of about $\frac{1}{3} \mathrm{~K} \mathrm{~min}^{-1}$. This procedure determined the $\Delta T_{\mathrm{T}}$ in which the sIA underwent

$\uparrow$ A recent (Gregov and Lawson 1972) in situ FLM study of tungsten bombarded with 150 to $450 \mathrm{eV} \mathrm{Ar}^{+}$ions at $63 \mathrm{~K}$ also suffers from the problem of the use of impure tungsten (General Electric 218 wire--99.95\% purity). $\ddagger$ The specimen failure rate for this experiment was high, as it was found to be difficult to perform field evaporation experiments on platinum in the $10-15 \mathrm{~K}$ temperature range without the occurrence of slip.

$\S$ In the case of platinum the temperature ranges of stages I to III are as follows: stage 1,0 to $35 \mathrm{~K}$; stage II, 35 to $500 \mathrm{~K}$; and stage III, 500 to $700 \mathrm{~K}$ (see Duesing and Schilling 1969, Dibbert et al 1971 and Doyama et al 1971). 
long range migration and hence established an isochronal recovery spectrum. The composite plot of the fraction of the total number of SIA per $2 \mathrm{~K}$ temperature interval against $T$ is shown in figure 5. The total number of SIA observed for this experiment was 93. All the SIA detected in these experiments exhibited a simple single extra bright spot type of contrast. The envelope of the experimental histogram shows a peak at about $14 \mathrm{~K}$ and that the recovery of SIA in substage $\mathrm{I}_{\mathrm{E}}$ was finished by $27 \mathrm{~K}$. It is emphasized that all the observed recovery was due to a substage $\mathrm{I}_{\mathrm{E}}$ SIA undergoing uncorrelated long range migration.

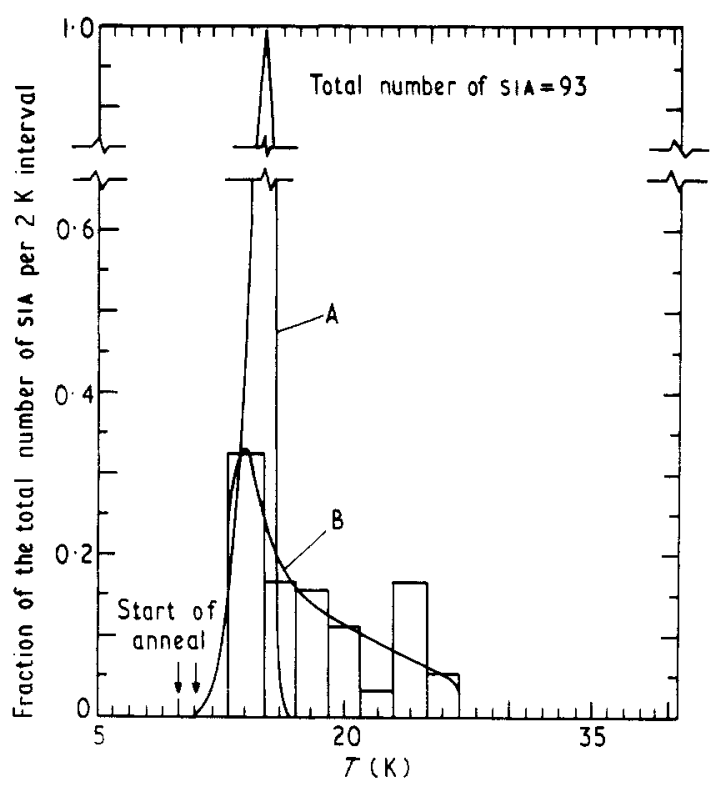

Figure 5. The composite spectrum for the isochronal recovery of seven platinum specimens which were irradiated at 10 or $11 \mathrm{~K}$ with 20 or $30 \mathrm{keV} \mathrm{Pt}^{+}$ions and then warmed at an average linear rate of $\frac{1}{3} \mathrm{~K} \mathrm{~min}^{-1}$ to $76 \mathrm{~K}$. The calculated peak shape for $\Gamma=10^{17}(\mathrm{~A})$ is superimposed on the experimental data so that the area under the calculated curve is equal to the area under the approximate envelope $(B)$ of the experimental histogram.

\section{A diffusion model for substage $I_{F}$ applicable to the FIM isochronal recovery experiments}

\subsection{The model}

The model presented in this section allowed the determination of an enthalpy of migration $\left(H_{1 \mathrm{i}}^{\mathrm{m}}\right)$, the diffusivity $\left(D_{1 \mathrm{i}}\right)$ and a range of limiting values of the volume of migration of $\left(V_{1 \mathrm{i}}^{\mathrm{m}}\right)$ of a SIA from the observed recovery spectra. The model was based on the following assumptions: (i) the FIM tip was approximated by a sphere of radius $R$; (ii) the initial distribution of SIA was uniform; (iii) the specimen's surface was the main sink for SIA; (iv) there were no SIA clusters or SIA impurity atom clusters $\dagger$ formed during isochronal warming; (v) the diffusion of SIA occurred via a single thermally activated process; and (vi) the concentration gradient had no angular dependence. Scanlan et al (1971b) have shown that the effect of a nonuniform distribution of SIA on the calculated recovery

$\dagger$ The case with SIA impurity atom clusters is considered in $\$ 5$. 
spectrum is negligible, hence justifying assumption (iii). In addition, both Scanlan et al (1971b) and Petroff and Seidman (1972a) have demonstrated that the distribution of the number of SIA per equal element of area on the surface of the specimens was not a function of the angular position at which the sIA emerged at the surface, thus vindicating assumption (vi).

The observable quantity in the isochronal recovery experiments was the flux of SIA (number $\mathrm{s}^{-1}$ ) crossing the surface of a specimen as it was warmed from $T_{0}$. The flux at any point $J_{1 \mathrm{i}}^{*}$ inside the specimen is given by

$$
J_{1 \mathrm{i}}^{*}(p, T(t))=-D_{1 \mathrm{i}}(p, T(t)) \nabla c_{1 \mathrm{i}}-\frac{c_{1 \mathrm{i}} \bar{V}_{1 \mathrm{i}}}{k T(t)} D_{1 \mathrm{i}}(p, T(t)) \nabla p
$$

where $p$ is pressure, $T(t)$ is the experimental expression for the warming rate, $c_{1 \mathrm{i}}$ the concentration of SIA, $\bar{V}_{1 \mathrm{i}}$ is the partial atomic volume of the SIA, $\nabla p$ the pressure gradient and $k$ is Boltzmann's constant. Application of the equation of continuity to equation (1) under the assumption of an isotropic linear elastic solid and hydrostatic pressure yields the governing equation (Johnson 1970)

$\frac{\partial c_{1 \mathrm{i}}}{\partial t}=D_{1 \mathrm{i}}(p, T(t))\left\{\nabla^{2} c_{1 \mathrm{i}}+\frac{\left(\bar{V}_{1 \mathrm{i}}-V_{1 \mathrm{i}}^{\mathrm{m}}\right)}{k T(t)} \nabla p \cdot \nabla c_{1 \mathrm{i}}-\left(\bar{V}_{1 \mathrm{i}} V_{1 \mathrm{i}}^{\mathrm{m}}\right)\left(\frac{\nabla p}{k T(t)}\right)^{2} c_{1 \mathrm{i}}\right\}$

Pétroff and Seidman (1972a) have presented semiquantitative arguments which demonstrated that the values of $|\nabla p|$ in the tip region $\dagger$ are not large enough to make the kinetic term $-\bar{V}_{1 \mathrm{i}} V_{1 \mathrm{i}}^{\mathrm{m}}(\nabla p / k T(t))^{2} c_{1 \mathrm{i}}$ and the drift term $\left(\bar{V}_{1 \mathrm{i}}-V_{1 \mathrm{i}}^{\mathrm{m}}\right) \nabla p \cdot \nabla c_{1 \mathrm{i}} / k T(t)$ significant in comparison to the first term on the right hand side of equation (2). Hence, the governing diffusion equation became

$$
\frac{\partial c_{1 \mathrm{i}}}{\partial t}=D_{1 \mathrm{i}}(p, T(t))\left\{\frac{\partial^{2} c_{1 \mathrm{i}}}{\partial r^{2}}+\frac{2}{r}\left(\frac{\partial c_{1 \mathrm{i}}}{\partial r}\right)\right\}
$$

which was solved subject to the boundary conditions

$$
\begin{array}{ll}
c_{1 \mathrm{i}}=c_{1 \mathrm{i}}^{0} & \text { all } r \text { at } t=0 \\
c_{1 \mathrm{i}}=0 & \text { at } r=R \text { for } t>0 .
\end{array}
$$

The solution for the flux of SIA crossing a specimen's surface $\left(J_{1 \mathrm{i}}\right)$ as a function of $T$ is

$$
J_{1 \mathrm{i}} \simeq \beta \exp \left(-\frac{1}{y}\right) \sum_{n=1}^{\infty} \exp \left\{-n^{2} \Gamma y^{2} \exp \left(-\frac{1}{y}\right)\right\}
$$

where $y$ is $k T / H_{1 \mathrm{i}}^{\mathrm{m}}, \Gamma$ is $\pi^{2} D_{1 \mathrm{i}}^{0} H_{1 \mathrm{i}}^{\mathrm{m}} / R^{2} \alpha k$ (these two parameters are dimensionless) and $\beta$ is $\xi 8 \pi R c_{1 \mathrm{i}}^{0} D_{1 \mathrm{i}}^{0}$. The quantity $D_{1 \mathrm{i}}^{0}$ is the pre-exponential factor of the SIA diffusion coefficient, $c_{1 \mathrm{i}}^{0}$ the initial concentration of SIA (number $\mathrm{cm}^{-3}$ ) and $\xi$ is the fraction of the total area of the sphere which was observed. In figure $6 J_{1 i} / \beta$ is plotted as a function of $y$ for $\Gamma=10^{17}$. The curve labelled 'no impurity atoms' is the one relevant to the present discussion. The shape of the peak is asymmetric and is narrow with a width in $\mathrm{K}$ at half maximum which is given by the expression

$$
\Delta T\left\{\left(\frac{J_{1 \mathrm{i}}}{\beta}\right)_{m / 2}\right\}=0.335 \times 10^{-2}\left(\frac{H_{1 \mathrm{i}}^{\mathrm{m}}}{k}\right) .
$$

+ A salue of $|\nabla p|$ was calculated from the average normal stress function presented by Smith and Smith (1970) for a FIM tip subjected to an electric field of $4.5 \mathrm{~V}(\AA)^{-1}$. 
The maximum occurs at a temperature $T_{\mathrm{m}}$ which is given by the approximate expression

$$
T_{\mathrm{m}} \simeq \frac{H_{1 \mathrm{i}}^{\mathrm{m}}}{k}(\ln \Gamma-2 \ln \ln \Gamma)^{-1} .
$$

The fractional error in the position of $T_{\mathrm{m}}$ for an uncertainty in $\Gamma$ is given by the equation

$$
\frac{\mathrm{d} T_{\mathrm{m}}}{T_{\mathrm{m}}} \simeq \frac{-(2-\ln \Gamma)}{\ln \Gamma(\ln \Gamma-2 \ln \ln \Gamma)}\left(\frac{\mathrm{d} \Gamma}{\Gamma}\right) .
$$

Hence small variations in $\alpha$ and $R$ from specimen to specimen could not have caused a significant variation in $T_{\mathrm{m}}$ and justified combining all the annealing spectra from different specimens for a given metal into one composite spectrum.

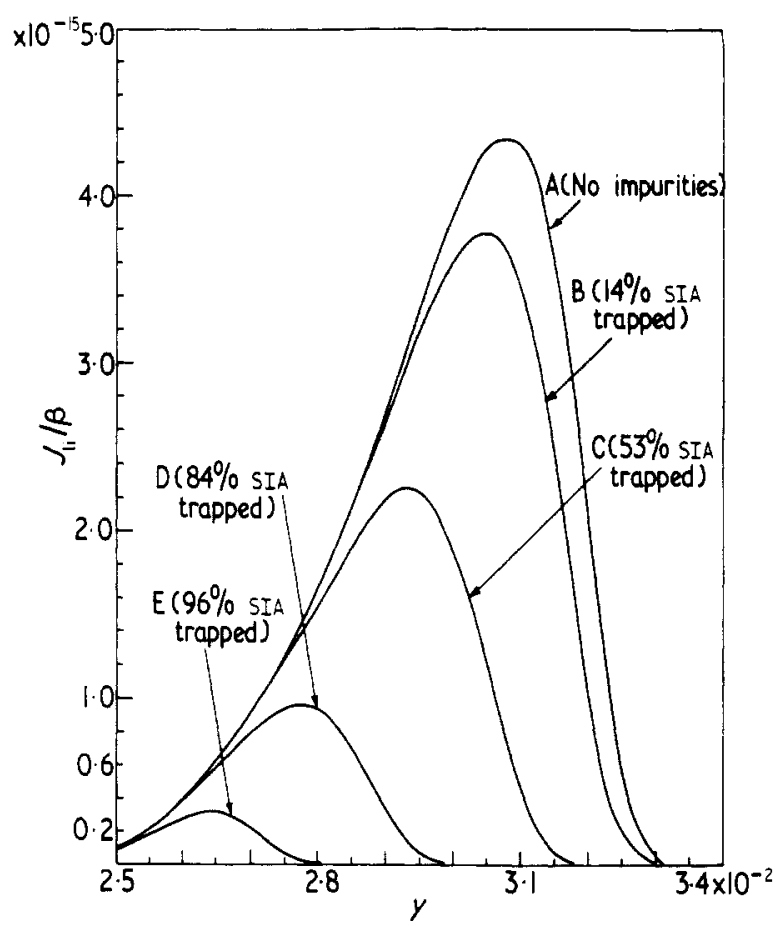

Figure 6. A plot of $J_{1 i} / \beta$ against $y$ for different concentrations of impurity atoms $\left(c^{*}=0,10^{-6}\right.$, $10^{-5}, 10^{-4}$ and $10^{-3}$ at fr; curves, A B, C, D and E respectively). The quantity $J_{1 \mathrm{i}}$ is the flux of SIA which crosses the surface of an FIM specimen during an isochronal anneal. The parameters $J_{1 i} / \beta, y, \Gamma\left(=10^{17}\right), \gamma(=0.025), \delta\left(=1.75 \times 10^{4}\right)$ and $H_{\mathrm{iv}}^{\mathrm{b}} / H_{1 \mathrm{i}}^{\mathrm{m}}(=1,2$ and 10$)$ are all dimensionless quantities. See $\S 4.1$ and $\S 5$ for the definitions of these parameters.

The calculated peak shape (equation (3)) was superimposed on a spectrum by matching the first inflection point of the approximate envelope of the experimental histogram with the first inflection point of the calculated peak. The superposition was performed so that the area under the calculated peak was equal to the area under the envelope of the experimental histogram. This procedure yielded a value of $T_{\mathrm{m}}$ which was then used to calculate $H_{1 \mathrm{i}}^{\mathrm{m}}$ and finally $D_{1 \mathrm{i}}^{\circ}$ was calculated from the expression for $\Gamma$. The value of $H_{1 \mathrm{i}}^{\mathrm{m}}$ calculated by this procedure was not very sensitive to the value of $\Gamma$ employed but the calculated value of $D_{1 \mathrm{i}}^{0}$ was almost linearly proportional to the value of $\Gamma$ employed, hence the values of $D_{1 i}^{0}$ reported are uncertain by a factor of approximately 10 . 
Since the FIM tip is subjected to a value of $p$ which is given by $E^{2} / 8 \pi$ (for a spherical tip) the quantity $H_{1 \mathrm{i}}^{\mathrm{m}}$ is related to the internal energy of migration $\left(U_{\mathrm{ij}}^{\mathrm{m}}\right)$ of an SIA by the thermodynamic equation

$$
H_{1 \mathrm{i}}^{\mathrm{m}}=U_{1 \mathrm{i}}^{\mathrm{m}}-\frac{E^{2}}{8 \pi} V_{1 \mathrm{i}}^{\mathrm{m}} .
$$

If the $E^{2} V_{1 \mathrm{i}}^{\mathrm{m}} / 8 \pi$ term in equation (4) is a significant fraction of $C_{1 \mathrm{i}}^{\mathrm{m}}$, then the position at which $T_{\mathrm{m}}$ occurs for the FIM isochronal recovery experiments will be less than the $T$ at which the maximum occurs for the substage $I_{E}$ peak as determined by resistivity measurements. For a pure hydrostatic pressure the shape of the annealing peak (equation (3)) is independent of the magnitude of $E^{2} V_{1 \mathrm{i}}^{\mathrm{m}} / 8 \pi$ but the width in $\mathrm{K}$ at half maximum is decreased.

\subsection{Application to the FIM tungsten recovery spectrum}

The superposition of equation (3) on the experimental recovery spectrum is shown in figure $3(b)$ for $\Gamma=10^{16}$. The values of $H_{1 \mathrm{i}}^{\mathrm{m}}$ and $D_{1 \mathrm{i}}^{0}$ obtained for $\Gamma=10^{16}$ are about $0.085 \mathrm{eV}$ and about $0.22 \mathrm{~cm}^{2} \mathrm{~s}^{-1}$. For $\Gamma=10^{15}$ the quantity $D_{1 \mathrm{i}}^{0}$ is $0.025 \mathrm{~cm}^{2} \mathrm{~s}^{-1}$, and $H_{1 \mathrm{i}}^{\mathrm{m}}$ is $0.079 \mathrm{eV}$; therefore, we concluded that a set of reasonable values for these quantities is $H_{1 \mathrm{i}}^{\mathrm{m}} \simeq 0.08 \mathrm{eV}$ and $D_{1 \mathrm{i}}^{0} \simeq(0.03$ to 0.2$) \mathrm{cm}^{2} \mathrm{~s}^{-1}$.

The calculation of $V_{1 \mathrm{i}}^{\mathrm{m}}$ via equation (4) required a value of $U_{1 \mathrm{i}}^{\mathrm{m}}$ for substage $\mathrm{I}_{\mathrm{E}}$ of tungsten. The main problem here was that the seven different irradiation studies on the stage I recovery behaviour have produced rather different recovery spectra. Figure 2 in the article by Kunz et al (1972) compares their recovery spectrum with the spectra determined by Burger et al (1966), Coltman et al (1967), Neely et al (1968) and DiCarlo et al (1969)†. An examination of this figure shows that the agreement among the various groups is poor. To complicate matters Kunz et al (1972) have demonstrated that the effect of irradiating a specimen which had been prequenched prior to the irradiation was to enhance recovery in five of the six peaks they observed in stage I (see their figure 5). If Kunz et al (1972) had quenched in vacancies then, in principle, only the substage $\mathrm{I}_{\mathrm{E}}$ recovery should have been enhanced. Thus, it is still uncertain as to which peak, from among the available results, in stage I should be labelled $\mathrm{I}_{\mathrm{E}}$. A much needed study is a resistivity recovery experiment on electron irradiated specimens for $\mathscr{R}$ values greater than $(4$ to 5$) \times 10^{4}$, as all of the work prior to the Kunz et al investigation was performed on tungsten with an $\mathscr{R}$ value of less than or approximately equal to $3 \times 10^{3}$. In the Kunz et al work the value of $\mathscr{R}$ was about 12000 . In view of this complicated situation we have arbitrarily taken $U_{1 \mathrm{i}}^{\mathrm{m}}=0.17 \mathrm{eV}$ (the $70 \mathrm{~K}$ peak of Neeley et al 1968) and calculated $V_{1 \mathrm{i}}^{\mathrm{m}}$ from equation (4) for $E=5 \mathrm{~V} \AA^{-1}$. The result is that $V_{1 \mathrm{i}}^{\mathrm{m}}<0 \cdot 1 \Omega_{\mathrm{a}}(\mathrm{W})_{+}^{+}$. This small value of $V_{1 \mathrm{i}}^{\mathrm{m}}$ is what makes it possible to observe the SIA at a high value of $E$. Note that if $V_{1 \mathrm{i}}^{\mathrm{m}}$ were as large as $0.65 \Omega_{\mathrm{a}}(\mathrm{W})$ then for $E=5 \mathrm{~V} \AA^{-1}$ and $U_{1 \mathrm{i}}^{\mathrm{m}}=0.17 \mathrm{eV}$ the value of $H_{1 \mathrm{i}}^{\mathrm{m}}$ would be zero and the application of $E$ would cause all the sIA to diffuse out of the specimens at the irradiation temperature.

The upper limit of $0 \cdot 1 \Omega_{\mathrm{a}}(\mathrm{W})$ for $V_{1 \mathrm{i}}^{\mathrm{m}}$ is in good agreement with the available values for this quantity calculated by Johnson $(1964 a, 1964 b)$. He determined that in the case of $\alpha$ iron $V_{1 \mathrm{i}}^{\mathrm{m}}$ is $0 \cdot 1 \Omega_{\mathrm{a}}(\alpha \mathrm{Fe})$ for the $\langle 110\rangle$ split SIA and $00 \Omega_{\mathrm{a}}(\alpha \mathrm{Fe})$ for the $\langle 111\rangle$ crowdion.

+ The sixth study is an unpublished electron irradiation experiment by Roberts (1968a, 1968b). The seventh study is a fast neutron irradiation experiment by Takamura et al (1971).

$\ddagger$ The quantity $\Omega_{\mathrm{a}}(\mathrm{W})$ is the atomic volume of a tungsten atom. A similar notation is employed for the other metals discussed. 


\subsection{Application to the FIM platinum recovery spectrum}

The peak shape for $\Gamma=10^{17}$ is superimposed on the experimental platinum isochronal recovery spectrum in figure 5 . This superposition implied that $T_{\mathrm{m}}=15 \mathrm{~K}$ and consequently that $H_{1 \mathrm{i}}^{\mathrm{m}} \simeq 0.042 \mathrm{eV}$ and $D_{1 \mathrm{i}}^{0} \simeq 0.77 \mathrm{~cm}^{2} \mathrm{~s}^{-1}$. For $\Gamma=10^{16}$ the values of these quantities are $H_{1 \mathrm{i}}^{\mathrm{m}} \simeq 0.0395 \mathrm{eV}$ and $D_{1 \mathrm{i}}^{0} \simeq 0.083 \mathrm{~cm}^{2} \mathrm{~s}^{-1}$. Hence, a set of best values for the SIA in substage $\mathrm{I}_{\mathrm{E}}$ is $H_{1 \mathrm{i}}^{\mathrm{m}} \simeq 0.04 \mathrm{eV}$ and $D_{1 \mathrm{i}}^{0} \simeq(0.08$ to 0.77$) \mathrm{cm}^{2} \mathrm{~s}^{-1}$.

The only other values of $D_{1 \mathrm{i}}^{0}$ reported for an SIA in platinum are those given by Sonnenberg et al (1971). They reported a range of possible values for $D_{1 \mathrm{i}}^{0} / a^{2}\left((1 \cdot 1\right.$ to $\left.3 \cdot 4) \times 10^{12} \mathrm{~s}^{-1}\right)$ which were consistent with both their experimental and theoretical isochronal recovery curves. Our range of values for $D_{1 \mathrm{i}}^{0} / a^{2}(a=2 \cdot 72 \AA)$ for $\Gamma$ equal to $10^{16}-10^{17}$ is $(1 \cdot 1$ to 10$) \times$ $10^{14} \mathrm{~s}^{-1}$, which differ from the Sonnenberg et al values by a factor of about (33 to 100). Sonnenberg et al consider their values of $D_{1 \mathrm{i}}^{0} / a^{2}$ to be uncertain by a factor ten, hence it is clear that the two sets of values for $D_{1 \mathrm{i}}^{0}$ can be made to overlap.

Sonnenberg et al (1971) have measured a value of $U_{1 \mathrm{i}}^{\mathrm{m}}=0.063 \mathrm{eV}$ for substages $\mathrm{I}_{\mathrm{D}}$ and $\mathrm{I}_{\mathrm{E}}$ in platinum. The evaporation field of platinum at $0 \mathrm{~K}$ is about $4.75 \mathrm{~V} \AA^{-1}$ (Müller and Tsong 1969). Since the BIF employed at 10 or $11 \mathrm{~K}$ is less than about $4.75 \mathrm{~V} \AA^{-1}$, we took $E$ to be between 4.75 and $4 \mathrm{~V} \AA^{-1}$. This implied that $V_{\mathrm{li}}^{\mathrm{m}}$ was in the range $(2.3$ to $3.2) \times 10^{-2} \Omega_{\mathrm{a}}(\mathrm{Pt})$. The only calculation of $V_{1 \mathrm{i}}^{\mathrm{m}}$ for a sIA in a fcc metal is for the $\langle 100\rangle$ split SIA in nickel (Johnson 1966) and a value of $0.1 \Omega_{\mathrm{a}}(\mathrm{Ni})$ was obtained. The value of $E^{2} V_{1 \mathrm{i}}^{\mathrm{m}} / 8 \pi$ for $0.1 \Omega_{\mathrm{a}}$ is between about $(0.066$ to 0.092$) \mathrm{eV}$. Thus, it is clear that if $V_{1 \mathrm{i}}^{\mathrm{m}}$ were as large as $0 \cdot 1 \Omega_{\mathrm{a}}(\mathrm{Pt})$, the quantity $H_{1 \mathrm{i}}^{\mathrm{m}}$ would have been negative under our experimental conditions.

\section{An impurity delayed diffusion model for substage $I_{E}$ applicable to FIM isochronal recovery experiments}

In the model presented in $\$ 4.1$ it was assumed that there were no SIA impurity atom clusters formed. It is known that small concentrations of impurity atoms reduce the fractional amount of recovery in $\mathrm{I}_{\mathrm{E}}$ (eg see Sosin 1967 and Schilling et al 1970). Hence, we considered (Lie and Seidman, to be published) the effect of the presence of impurity atoms on the flux of SIA crossing an FIM specimen's surface which is warmed through substage $I_{E}$.

We employed diffusion theory that was an extension of the impurity delayed diffusion model developed by Sosin and Bauer (1966). The coupled linear partial differential equations governing the situation are

$$
\begin{aligned}
& \frac{\partial c_{1 \mathrm{i}}}{\partial t}=D_{1 \mathrm{i}}(p, T(t)) \nabla^{2} c_{1 \mathrm{i}}-\lambda(T(t)) c_{1 \mathrm{i}}+\mu(T(t)) c_{\mathrm{i}} \\
& \frac{\partial c_{\mathrm{i}}}{\partial t}=\lambda(T(t)) c_{1 \mathrm{i}}-\mu(T(t)) c_{\mathrm{i}}
\end{aligned}
$$

where $c_{\mathrm{i}}$ is the concentration of SIA impurity atom complexes. Equations (5) and (6) are linear because it was assumed that the concentration of impurity atoms was a constant. The trapping of SIA by impurity atoms was represented by $-\lambda(T(t)) c_{1 \mathrm{i}}$ and the release of SIA from the immobile SIA impurity atom complexes by $\mu(T(t)) c_{\mathrm{i}}$ in equation (5). The rate constants $\lambda(T(t))$ and $\mu(T(t))$ are defined by

$$
\lambda=g_{1} c^{*} v \exp \left\{\frac{-G_{\mathrm{li}}^{\mathrm{m}}}{k T(t)}\right\}=\lambda^{0} \exp \left\{\frac{-H_{\mathrm{li}}^{\mathrm{m}}}{k T(t)}\right\}
$$




$$
\mu=g_{2} v \exp \left\{\frac{-\left(G_{1 \mathrm{i}}^{\mathrm{m}}+G_{1 \mathrm{i}}^{\mathrm{b}}\right)}{k T(t)}\right\}=\mu^{\mathrm{o}} \exp \left\{\frac{-\left(H_{1 \mathrm{i}}^{\mathrm{m}}+H_{1 \mathrm{i}}^{\mathrm{b}}\right)}{k T(t)}\right\}
$$

where $c^{*}$ is the concentration of immobile impurity atoms, $v$ is an atomic frequency factor, $g_{1}$ the number of ways an SIA can combine with an impurity atom to form a complex, $g_{2}$ is the number of ways in which the complex can dissociate, $G_{1 \mathrm{i}}^{\mathrm{m}}$ the Gibbs free energy of migration of an SIA, $G_{1 \mathrm{i}}^{\mathrm{b}}$ the Gibbs free binding energy of an SIA to the complex and $H_{1 \mathrm{i}}^{\mathrm{b}}$ the binding enthalpy of the sIA to the complex. The pre-exponential factors $\lambda^{0}$ and $\mu^{0}$ are given by

$$
\begin{aligned}
& \lambda^{0}=g_{1} c^{*} v_{0} \\
& \mu^{0}=g_{2} v_{0} \exp \left(S_{1 \mathrm{i}}^{\mathrm{b}} / k\right)
\end{aligned}
$$

where $v_{0}$ is a frequency factor which includes a migration entropy term, and $S_{1 \mathrm{i}}^{\mathrm{b}}$ is the binding entropy of an SIA to a complex. The initial and boundary conditions employed in the solution of equations (5) and (6) were

$$
\begin{aligned}
& c_{1 \mathrm{i}}=c_{1 \mathrm{i}}^{0} \quad r<R \text { at } t=0 \\
& c_{1 \mathrm{i}}=0 \quad r=R \text { for all } t \\
& c_{\mathbf{i}}=0 \quad \text { all } r \text { at } t=0 .
\end{aligned}
$$

Figure 6 presents the results of the calculation for $\Gamma=10^{17}, \gamma=0.025, \delta=1.75 \times 10^{4}$, and the ratio $H_{1 \mathrm{i}}^{\mathrm{b}} / H_{1 \mathrm{i}}^{\mathrm{m}}$ for the values 1,2 and 10 . The dimensionless parameters $\gamma$ and $\delta$ are given by

and

$$
\gamma=R^{2} \lambda^{0} / \pi^{2} D_{1 \mathbf{i}}^{0}
$$

$$
\delta=R^{2} \mu^{0} / \pi^{2} D_{1 \mathrm{i}}^{O} .
$$

This figure shows that the dominant effect of increasing $c^{*}$ at constant values of the above parameters is to decrease the amount of recovery in substage $I_{E}$; that is, to increase $c_{i}$. The concentration of trapped SIA is independent of $H_{1 \mathrm{i}}^{\mathrm{b}} / H_{1 \mathrm{i}}^{\mathrm{m}}$ for values of this ratio which are greater than or equal to one, but it is a strong function of $c^{*}$. For $c^{*}=10^{-6}$ at $\mathrm{fr}$ $\left(\mathscr{R} \simeq 4 \times 10^{4}\right)$ the fraction of $c_{1 \mathrm{i}}^{0}$ trapped is $0 \cdot 14$, and for $c^{*}=10^{-3}$ at fr $(\mathscr{R} \simeq 40)$ the fraction of $c_{1 \mathrm{i}}^{0}$ trapped is 0.96 . Thus for $H_{1 \mathrm{i}}^{\mathrm{b}} / H_{1 \mathrm{i}}^{\mathrm{m}} \geqslant 1$ the value of $c^{*}$ has a much stronger effect on the fraction of $c_{1 \mathrm{i}}^{0}$ which become trapped than does $H_{1 \mathrm{i}}^{\mathrm{b}}$.

The results discussed above explain why Scanlan et al (1971a) observed a recovery peak at about $38 \mathrm{~K}$, whereas Sinha and Müller did not observe this low temperature long range migration peak. The Sinha-Müller observation can be understood if it is recalled that their value of $c^{*}$ was probably greater than $10^{-3}$ at fr $(\Re<40)$. Thus the flux of SIA they observed at $90 \mathrm{~K}$ most likely corresponded to the release of SIA from impurity atom traps.

\section{FIM contrast patterns produced by self interstitial atoms}

A first order model has been developed (Seidman and Lie 1972) to predict the possible contrast effects that a given geometric configuration of an SIA might produce in an FIM image. The concept of this model is now discussed and applied to a $\langle 110\rangle$ split SIA, an example illustrating the effects predicted is presented, and a critique is made of prior FIM experiments to identify the configuration of an SIA. 


\subsection{The concept of a constant pattern}

An immobile SIA which is embedded inside an FIM specimen, well below the surface, may be uncovered and eventually removed by the field evaporation of successive atomic planes. The long range dilatational field of the SIA results in the displacement of lattice atoms in and near the surface. This displacement of atoms results in three possible contrast effects which can be understood with the aid of a hard sphere model of a (111) plane (see figure 7). The number on each atom indicates the layer in which it resides. The

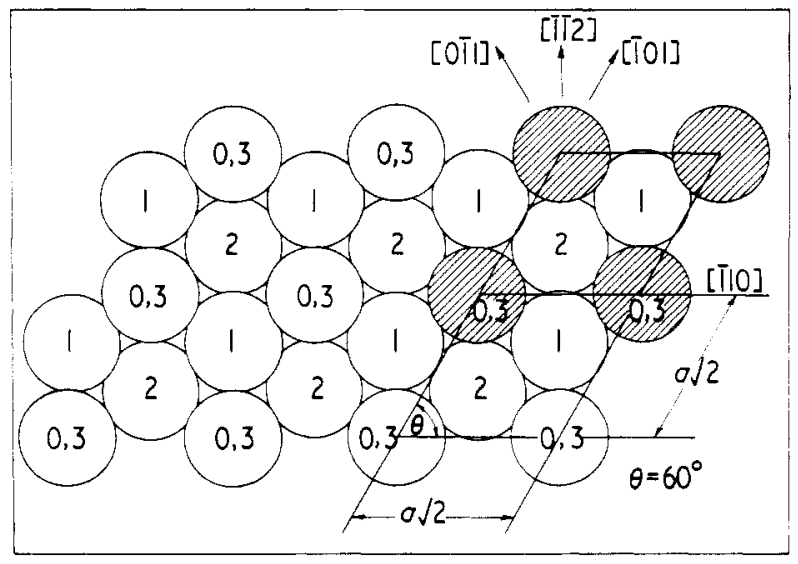

Figure 7. A hard sphere map of the arrangement of atoms in the (111) plane of a bcc lattice (the atomic diameter is to scale). The numbers labelling the atoms correspond to the plane in which the atoms reside. The number 0 is the surface plane, the number 1 is the plane which is one interplanar spacing down, etc.

surface layer is labeled 0 , and the first layer down 1, etc. The three possible contrast effects are as follows:

(i) A 'bright spot' is produced in the 0 layer if any one of the atoms in this layer is displaced normal to the (111) plane by a sufficient distance. The normal displacement causes an enhanced value of the local electric field $E_{1}$ at the site of an atom which results in an increased image intensity.

(ii) An 'extra bright spot' is produced in the 0 layer if an atom in plane number 1 is displaced normal to the (111) plane by an amount sufficient to raise $E_{1}$. The term 'extra bright spot' is employed since the atom being imaged is not one which resides in the 0 layer. This extra bright spot will increase in intensity as the atoms in the 0 layer are removed by field evaporation.

(iii) If the normal displacement of an atom is large enough to cause it to be preferentially field evaporated, then a bright spot or an extra bright spot can be replaced by a vacant site (a dark spot on a positive FIM micrograph). It is emphasized that the vacant sites are a direct result of the atomic displacements around an SIA and are not thermally generated vacancies, or vacancies produced by a bombarding particle.

All three of the above contrast effects may be produced by one sia. Thus, even though the extra atom itself may be unstable at the surface (Brandon 1968) it is still possible to detect an SIA via its long range dilatational field. The totality of all the contrast effects from a single SIA is termed a contrast pattern. The different possible geometric configurations (eg see Corbett 1966) of a SIA exhibit different contrast patterns on different planes. 
It is via the differences among the contrast patterns that it is possible to establish a set of selection rules from which the geometric configuration of the immobile SIA may be determined.

\subsection{Atomic displacement field of a $\langle 110\rangle$ split}

The atomic displacement field employed for the $\langle 110\rangle$ split sia was calculated by Beeler (private communication) for an $\alpha$ iron interatomic potential $\uparrow$ described by Johnson (1964a). No allowance was made for any surface relaxation effects. The total atomic displacement field was confined to three adjacent $\{110\}$ planes. 95 atoms were displaced for their normal atomic positions by greater than or equal to $10^{-2}$ of an interatomic

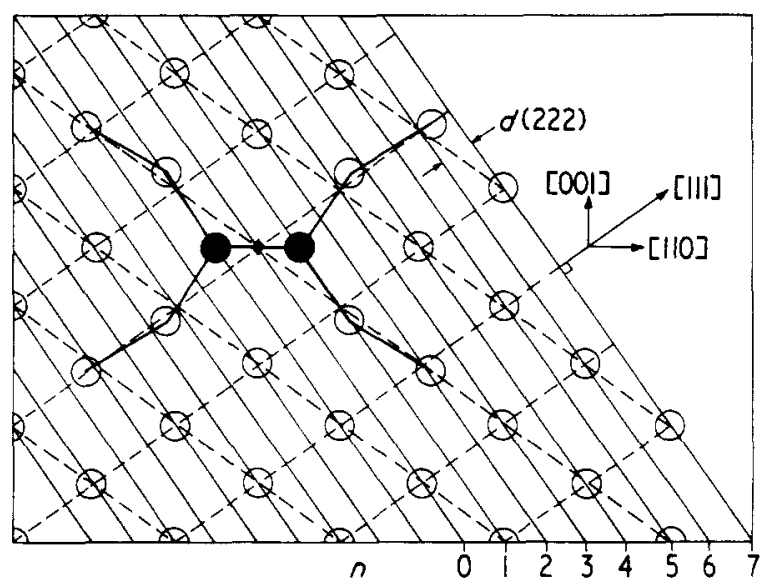

Figure 8. The atomic displacement field of the [110] split SIA in the (110) plane of $\alpha \mathrm{Fe}$ (bcc). The two solid black circles indicate the SIA configuration. The solid black diamond is the centre of mass of the configuration. The intersections of the broken lines are the centres of the atom positions in the perfect bcc lattice. The (1T0) plane is the central sheet (cs) of the atomic displacement field.

distance. Figure 8 shows the atomic displacements in the central (1110) plane around a [110] split SIA. The centre of mass (the black diamond) of this SIA is located seven (222) interplanar spacings (d(222)) below a (111) surface. The largest atomic displacements are for atoms which lie along the two $\langle 111\rangle$ directions in the $(1 \overline{10})$ plane. The atomic displacements in the front and back $(1 \overline{1} 0)$ planes are not shown but they are identical to one another in these two planes. The largest displacement of an atom in the central (110) plane is $0.388\left(\frac{1}{2} a_{0}\right)\left(a_{0}\right.$ is the lattice parameter) as compared to $0.111\left(\frac{1}{2} a_{0}\right)$ for an atom in the front or back $(1 \overline{1} 0)$ planes. This comparison excludes the lattice atom which is a member of the split configuration.

\footnotetext{
+ Benedek and Ho at Cornell University (private communication) have constructed a tungsten interatomic potential and calculated that the lowest energy configuration in tungsten is the $\langle 110\rangle$ split SIA. The atomic displacement field for the $\langle 110\rangle$ split SIA in tungsten is qualitatively similar to the one discussed for $\alpha$ iron. This result implies that the contrast effects suggested by the displacement field are not sensitive to the interatomic potential.
} 


\subsection{Atomic displacement patterns for the $\langle 110\rangle$ split SIA underneath the $\{111\}$ planes}

There are six nonequivalent orientations of the $\langle 110\rangle$ split SIA and it can be shown from symmetry considerations that only the $[110]$ and $[110]$ orientations produce different displacement patterns in the (111) planet. Thus, a consideration of these two $\langle 110\rangle$ orientations for the (111) plane give the entire group of displacement patterns for the $\langle 110\rangle$ split SIA underneath the $\{111\}$ planes.

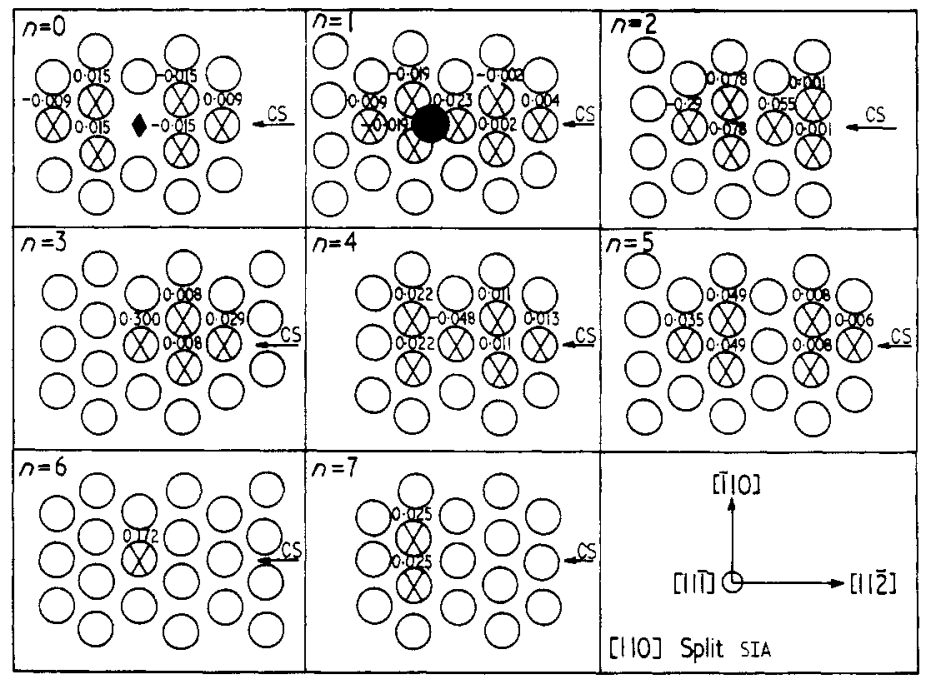

Figure 9. The (111) atomic displacement maps for the first seven planes above the centre of mass of the [110] split SIA in $\alpha \mathrm{Fe}$. The solid black diamond in the $n=0$ is the centre of mass of this configuration. The atoms with crosses inside them are the displaced ones, and the numbers associated with each atom are the normal displacements in units of $\left(a_{0} / 2\right)$. The arrows marked cs indicate the intersection of the cs of displaced atoms with the (111) plane

The displacement patterns in the first seven planes above the [110] split SIA are shown in figure 9 . The solid circle in $n=1$ is one member of the [110] split and the other member is in the $n=-1$ plane (not shown). The atoms marked with crosses are displaced from their perfect lattice sites and the number associated with each atom is the displacement normal to the (111) plane in units of $\frac{1}{2} a_{0}$ (note that $\mathrm{d}(222)$ is $(1 / \sqrt{ } 3)\left(\frac{1}{2} a_{0}\right)$ ). The letters Cs indicate the row of atoms in the (111) plane which intersects the central sheet of atoms containing the [110] split SIA.

We now consider the eight displacement patterns in figure 9 in detail. First note that there are both positive and negative normal displacements with respect to the (111) perfect lattice sites. 36 of the 38 displacements are very much less than $0 \cdot 1\left(\frac{1}{2} a_{0}\right)$. The two atoms with displacements greater than $0 \cdot 1\left(\frac{1}{2} a_{0}\right)$ are in the $n=6\left(0 \cdot 172\left(\frac{1}{2} a_{0}\right)\right)$ and $n=3$ $\left.(0.300)\left(\frac{1}{2} a_{0}\right)\right)(111)$ planes. Thus, if we only consider these two displaced atoms ${ }_{+}^{+}$, in addi-

$\dagger$ For a $\langle 110\rangle$ split S1A underneath the $\{h k l\}$ planes $(h \neq k \neq l)$ there are six different patterns for each $(h k l)$ plane, and for the $\{h k k\}$ planes $(h \neq k)$ there are four different patterns for each $(h k k)$ plane.

$\ddagger$ The consideration of only atoms with displacements greater than $0 \cdot 1\left(\frac{1}{2} a_{0}\right)$ is based on the results obtained by Moore $(1962,1968)$ and Moore and Ranganathan (1967) on computer simulated FiM images. They showed that an image could be simulated by retaining all atoms whose centres lay within a fixed distance of a spherical surface. The results in table 1 of the Moore and Ranganathan (1967) paper indicate that the neglect of the atoms with very small displacements $\left(\ll \frac{1}{2} a_{0}\right)$ from the $\{111\}$ planes was reasonable. 
tion to the [110] split SIA, the following constrast pattern might be expected during the course of the atom by atom dissection of successive (111) planes. The first contrast effect due to the sia might be observed as a weak extra bright spot in the $n=7$ plane due to the displaced atom in the $n=6$ plane. Once the $n=7$ plane had been field evaporated this displaced atom in the $n=6$ plane would most likely to be field evaporated preferentially and a vacant lattice site would be detected. If it were not immediately field evaporated preferentially, then this displaced atom would appear as a bright spot. Thus, it is possible to have a bright spot as well as an extra bright spot as a result of the normal displacement of an atom. Similarly, once the $n=4$ plane was reached an extra bright spot might be detected due to the displaced atom in the $n=3$ plane. Subsequently, this displaced atom might be preferentially field evaporated from the $n=3$ plane leaving behind a vacant lattice site. Finally, one member of the split sIA would be detected in the $n=1$ plane as an extra atom. After the $n=1$ plane had been field evaporated the vacant site in the $n=0$ plane would probably not be detected, as the other member (in the $n=-1$ plane) of the split SIA would relax into this site.

\subsection{Comparison with experimental results}

Figure 10 (plate) shows an example of a contrast pattern from a sia detected in a (111) plane of a tungsten specimen which had been irradiated with $20 \mathrm{keV} \mathrm{W}^{+}$ions at $18 \mathrm{~K}$ and then was examined immediately at this temperature. This $T$ is less than the value at which long migration occurs in tungsten therefore the SIA was immobile during the atom by atom dissection process. The 20 frames shown in figure 10 are from an $\sim 100$ frame sequence of $35 \mathrm{~mm}$ ciné film.

The schematic representation of the events occurring during the dissection process are indicated below each frame. The numbers $N=4$ to $N=0$ in the lower right hand corner of each schematic map indicate the number of the (111) plane field evaporated (the $N=4$ plane was the first plane field evaporated).

The first contrast effect detected was a weak bright spot (frame 2) in the $N=3$ plane. This weak bright spot persisted for a number of frames of film, and was finally field evaporated preferentially, leaving behind a vacant lattice site (frames 5 to 7 ) in the $N=3$ plane. The reason for this contrast effect must have been that the normal displacement of the atom from the $N=3$ plane was too small to cause an extra bright spot in the $N=4$ plane, thus the contrast effect consisted of only a bright spot followed by a vacant site. In frame 8 the dissection of the $N=2$ plane begins, and frame 13 is the last micrograph in this sequence. As the $N=2$ plane decreases in size a weak extra bright spot appears (frame 12) and this extra bright spot became brighter as atoms in the $N=1$ plane were removed (frames 13 to 17). This extra bright spot was finally field evaporated (frame 18) when the edge of the $N=1$ plane reached it. The $N=0$ plane was carefully scanned for contrast effects, but none were detected.

The above contrast pattern is a consequence of the long range atomic displacement field of a SIA. It is improbable that the bright spot and extra bright spot observed correspond to two different SIA, because of the low measured total sia concentration $\left(\sim 3 \times 10^{-4}\right.$ at fr) found in this specimen. Furthermore, it is also certain that the bright spot followed by the vacant site is not a Frenkel pair as the vacant site was observed to replace the bright spot. We cannot state definitely that this contrast pattem was due to a [110] split SIA as opposed to a [111] split crowdion.

In research in progress in this laboratory (Wilson and Seidman) we have found possible contrast patterns caused by SIA in the $\{221\},\{432\}$ and $\{332\}$ planes. Thus it is believed 
that all of the results obtained to date represent evidence for the validity of the concept of a contrast pattern.

\subsection{Discussion of other FIM research on SIA contrast effects}

On the basis of our model it is believed that the evidence published by Attardo et al (1967) for the existence of a $\langle 110\rangle$ split SIA in neutron irradiated tungsten is inadequate to prove their claim, even if this SIA is responsible for the stage III annealing peak. It is clear that the atom by atom dissection of high index planes is required in order to make any quantitative statements about the geometric configuration of the SIA. Since, they have not presented this detailed information their conclusion must be regarded with caution. The same cautionary statement applies to Attardo and Galligan's (1966a) claim that the stage III recovery peak in neutron irradiated platinum is caused by the motion of the $\langle 100\rangle$ split SIA.

In a recent study of ion implantation in iridium (O'Connor and Ralph 1972a, 1972b) a discussion was given of FIM contrast effects produced by SIA (O'Connor and Ralph 1972a). First, these authors suggested that the effect of the SIA should be observed once only for the SIA detected by the pulse field evaporation technique. Our experimental results indicate that this is not correct, as we have observed contrast patterns from a single SIA in both tungsten (Seidman and Lie 1972, Wilson and Seidman, unpublished) and platinum (Pétroff and Seidman 1972a). Second, they also assert that, 'only a few positions on the image are suitable for interstitial contrast, namely the plane ledges of low index poles whose ledge widths are relatively large'. We disagree with this statement for the following reasons:

(i) The atomic resolution in the low index regions of an FIM image is extremely poor when compared to the high index regions (eg compare the $\{111\}$ planes and the $\{110\}$ planes in a tungsten image). If only the low index regions are studied it negates almost completely the possibility of observing SIA contrast patterns.

(ii) The field evaporation characteristics of atoms from the ledges of low index planes produce 'bright spot' contrast effects which are not associated with SIA (eg see Buswell (1971) and Robinson et al (1972) for a discussion of Buswell's paper).

\section{Point defect structure of depleted zones}

\subsection{Experiment on irradiated tungsten}

We now describe some results (Beavan et al 1971) on the detailed point defect structure of depleted zones produced at low temperatures in tungsten which have been obtained with the FIM using the pulsed field evaporation technique.

The zones were produced in high purity tungsten $\left(\mathscr{R}>4 \times 10^{4}\right)$ by irradiation with $20 \mathrm{keV} \mathrm{W}^{+}$ions at $18 \mathrm{~K}$ and were then examined at the same temperature. The three dimensional structure of the positions of individual vacancies and SIA in the zones was derived from measurements made on the FIM ciné film (Scanlan et al 1969). The geometrical methods used to reconstruct the zones are described by Beavan et al (1971). The zone which we now consider was detected on a sequence of successive (111) planes located about $33 \AA$ below the original surface of the specimen surface which was exposed to the ion beam. Figure 11 (plate) shows 20 different micrographs of this zone taken at various stages of the dissection process which involved the recording and analysis of $4.4 \times 10^{3}$ 

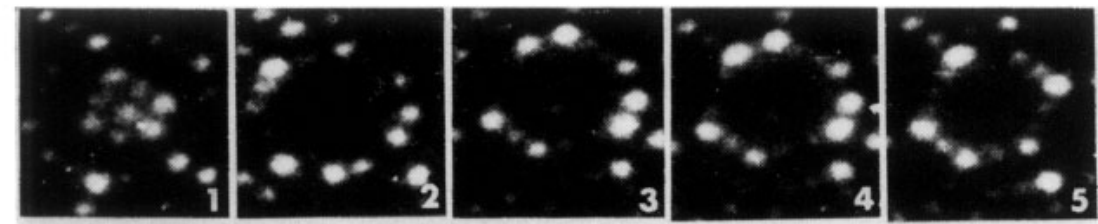

$$
N=4 \stackrel{-03}{*}
$$

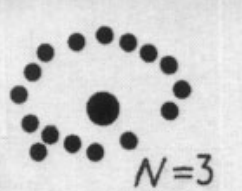

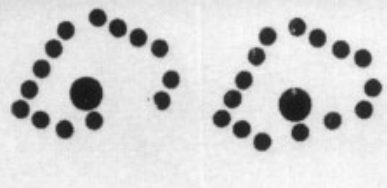
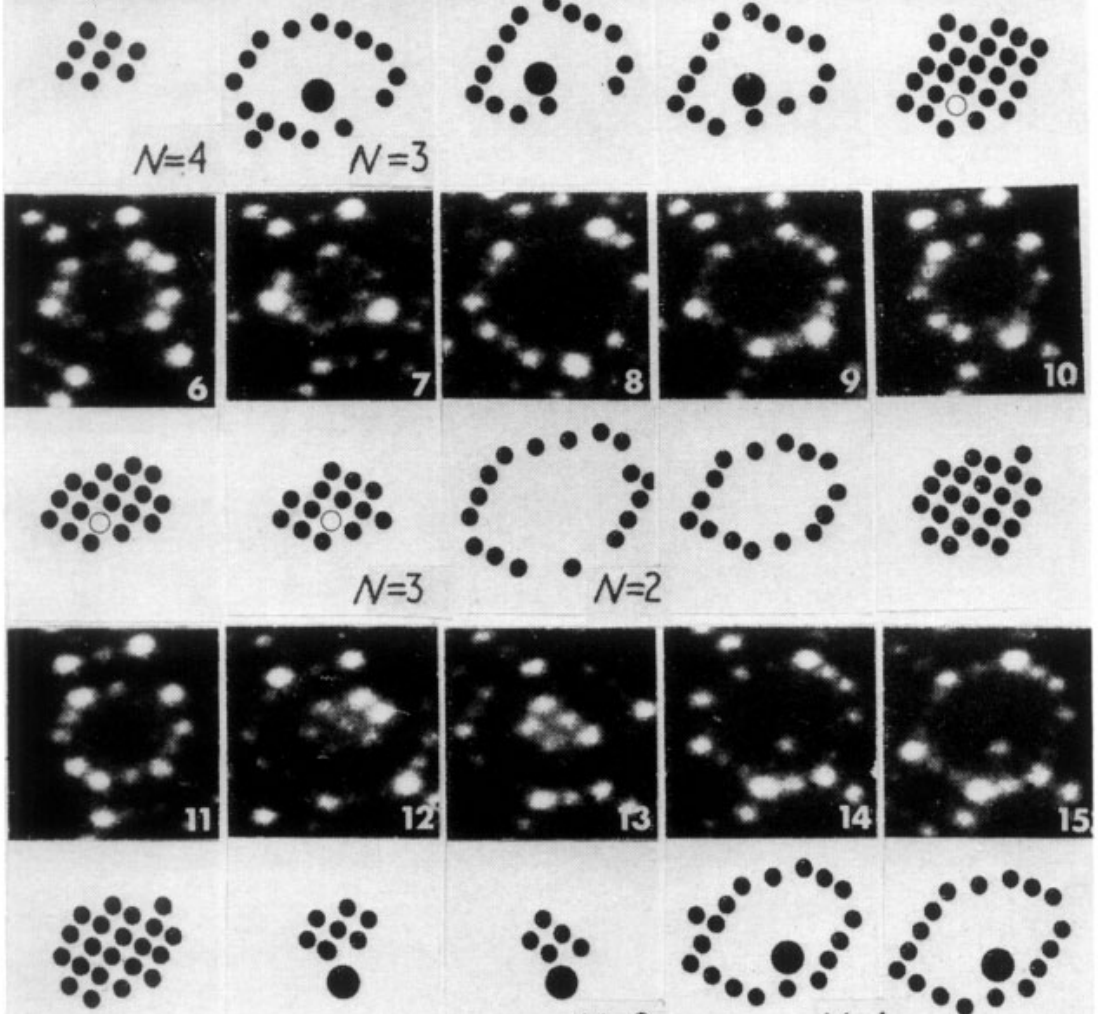

$$
N=2
$$
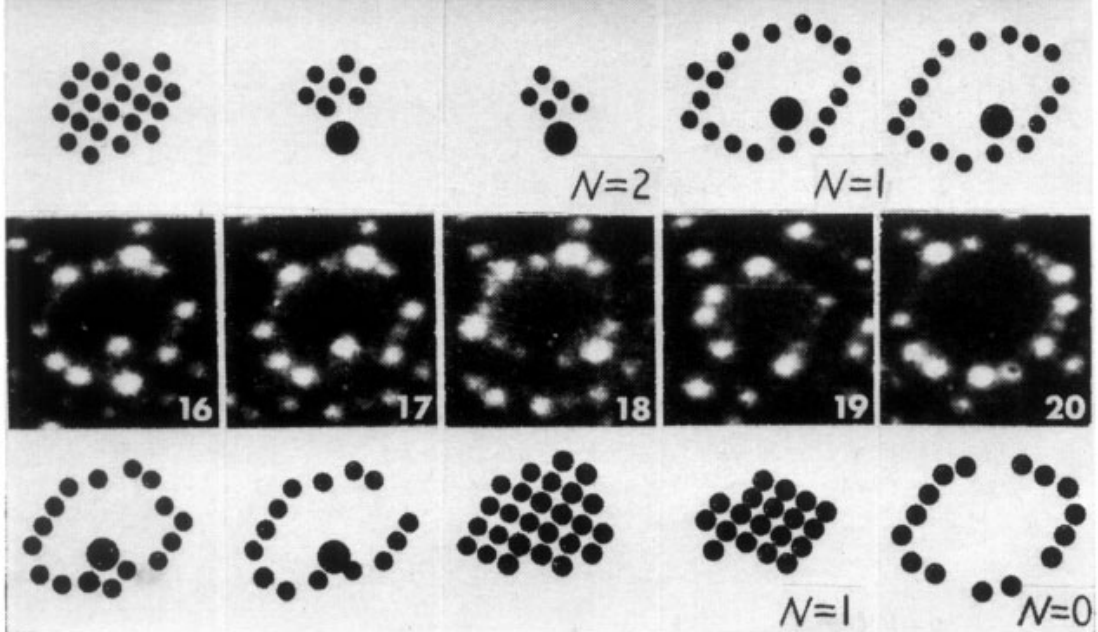

Figure 10. A 20 frame sequence out of a sequence of about 100 frames of $35 \mathrm{~mm}$ cine film showing the detection of a contrast pattern caused by a single sIA in a (111) plane of an irradiated tungsten specimen. This figure is discussed in detail in $\S 6.4$. 

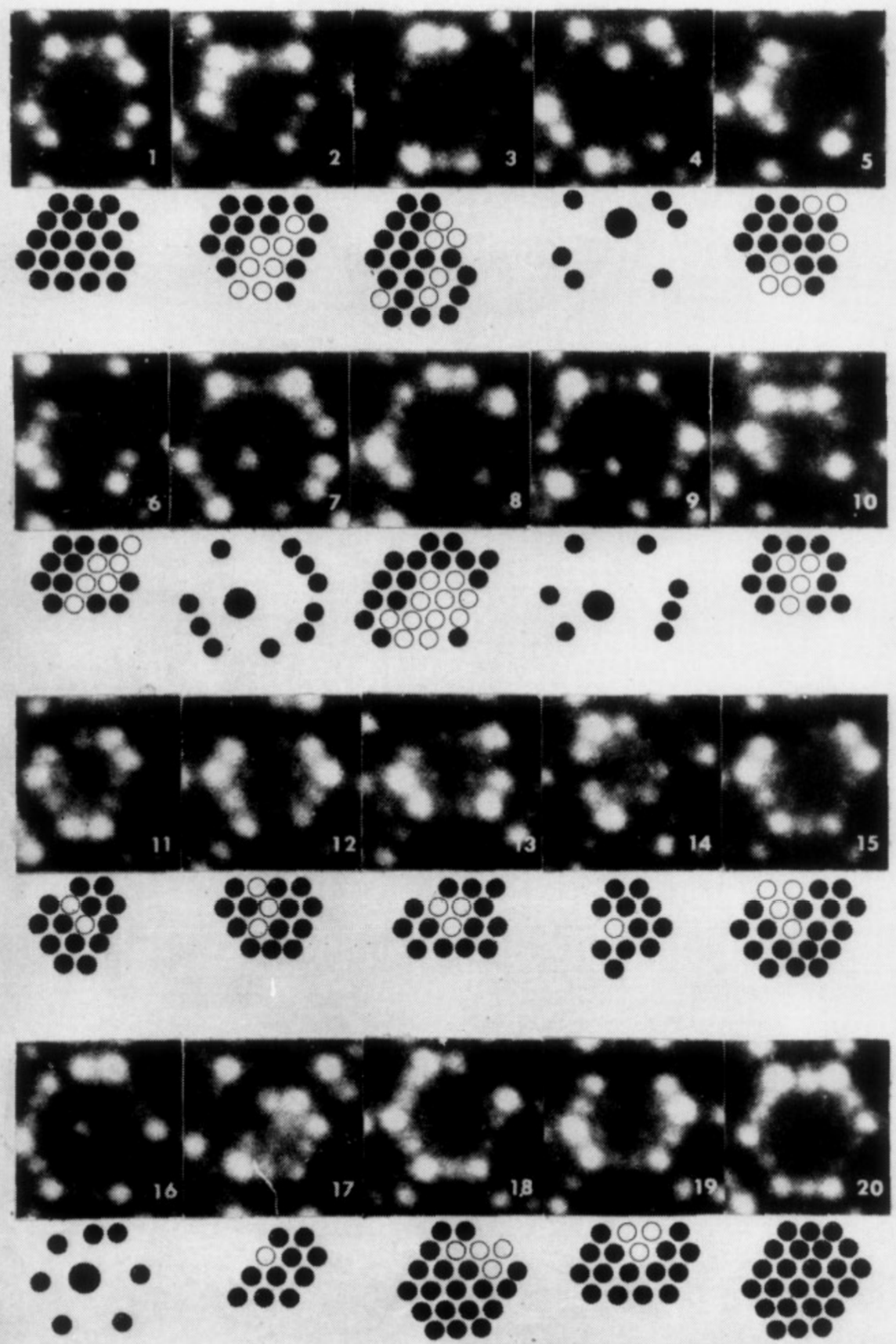

Figure 11. Twenty FIM micrographs, out of a sequence of $4.4 \times 10^{3}$ recorded frames, showing a depleted zone in tungsten detected in the (111) plane at various stages of the atom by atom dissection process. The larger solid black circles in frames $4,7,9$ and 16 indicate the SIA, the open circles indicate vacancies and the smaller solid black circles indicate lattice atoms 
frames of film. The positions of the atoms, sIA and vacancies found in this plane are illustrated schematically below each micrograph. In no case was any vacancy attributed to an apparent missing atom, unless the vacancy was certified visually in the FIM micrograph. The depleted zone appeared to be contained completely within the bounds of this stack of successive (111) planes, since no vacancies were found on any of the planes directly adjacent to it. In the entire volume of the specimen examined $\left(\sim 10^{-17} \mathrm{~cm}^{3}\right)$ this was the only depleted zone detected, hence we conclude that it was caused by a single incident ion.

There were 172 vacancies within the region (consisting of $\left.1850 \Omega_{\mathrm{a}}(\mathrm{W})\right)$ which contained the zone. In addition, 17 SIA were found in, or immediately adjacent to, the zone. This result yields an average SIA concentration of about $0.92 \mathrm{at} \%$. It has been shown (Seidman and Lie 1972) that a possible contrast effect accompanying the appearance of an siA is the observation of an artifact vacancy immediately prior to the detection of the SIA. Making this correction, the average concentration of vacancies in this zone was determined to be about 8.7 at \%. Thus, the depleted zone had a spongy character, and there was a large local imbalance between the SIA and vacancy concentrations.

Figure 12 shows a (111) projection of the vacancy and SIA positions. The vacancies are denoted by open circles, the sIA by solid triangles and the multiple concentric circles imply that more than one vacancy projected to the same atom position. This mode of projection reveals the three main branches of the zone. The direction of elongation of the three branches was along the three different $\langle 011\rangle$ directions lying in the (111)

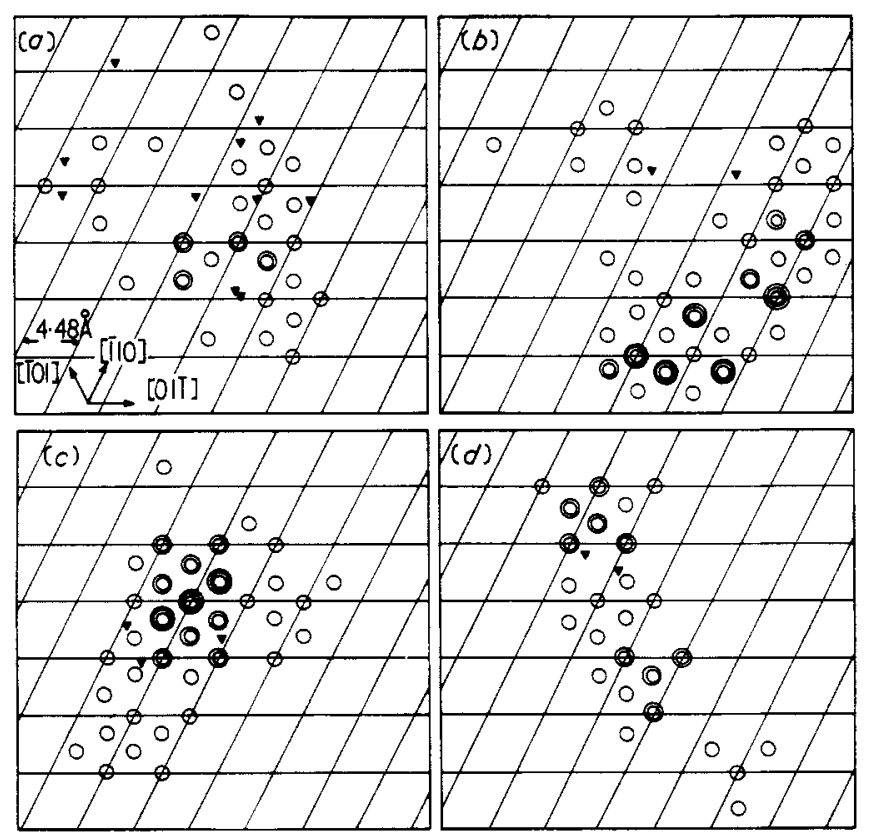

Figure 12. Four (111) projection maps of the vacancies and SIA found in the depleted zone shown in figure 11. (a) Projection of planes 1-10 onto 50:10 \$IA, 32 vacant sites. (b) projection of planes 11-20 onto 50; 2 SIA, 55 vacant sites. (c) Projection of planes 21-30 onto 50; 3 SIA, 50 vacant sites. (d) Projection of planes 31-50 onto 50:2 SIA, 35 vacant sites. The vacancies are indicated by open circles, the sia by solid black triangles and the multiple concentric circles imply that more than one vacancy projected to the same atomic position. Note the elongation of the depleted zone along the three different $\langle 110\rangle$ directions in the (111) plane. 


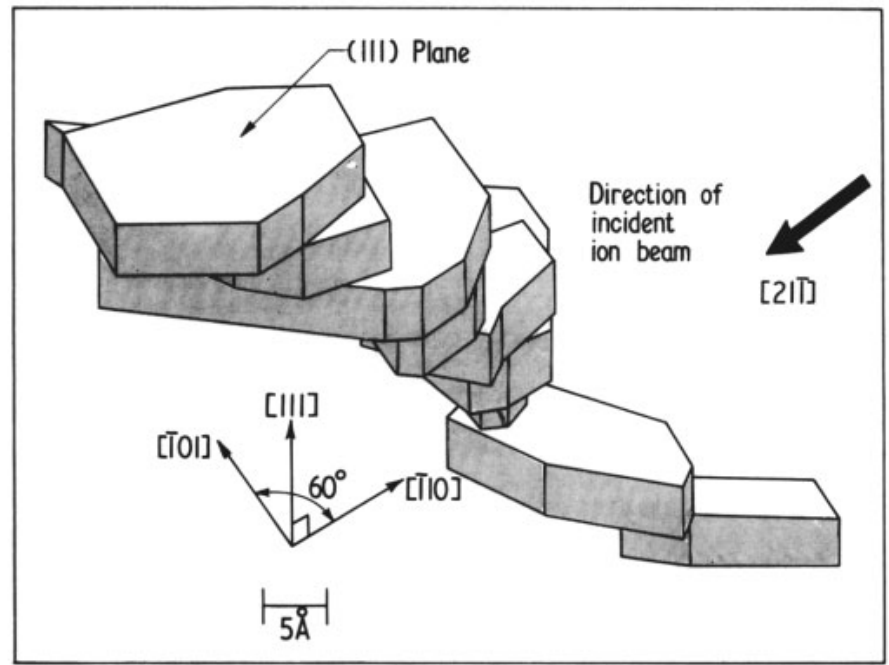

Figure 13. An isometric drawing of the depleted zone detected in the (111) plane constructed from the projection maps shown in figure 12 .

plane. An isometric drawing of this zone is shown in figure 13 where the thickness of each slab $\dagger$ is $4.56 \AA$ ( 5 atomic layers), and the boundary of each slab was determined by connecting the outer point defects on its periphery by straight line segments. Figures 11 to 12 show that the geometry of this zone is not sufficiently regular to allow it to be approximated by a sphere or a right circular cylinder, or for that matter, any simple geometric form.

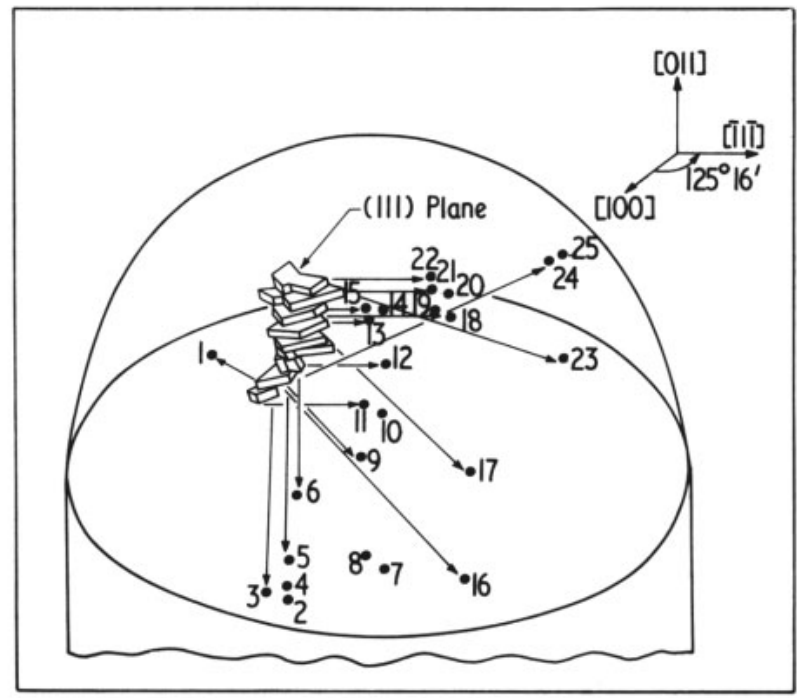

Figure 14. An isometric drawing showing the relationship of the SIA in the lattice to the position of the depleted zone detected in the (111) plane (figures 11 to 13). The direction of the incident ion beam $(\sim[211])$ is almost normal to the plane of the page. The arrows from the depleted zone to the sIA indicate the most probable propagation directions.

$\dagger$ With the exception of the third slab from the bottom, which is 10 atomic layers thick. 
A total of 25 additional SIA were found in the surrounding lattice at appreciable distances from the zone. The positions of these SIA are shown in figure 14. It was possible to show that 23 of these 25 sIA could have been propagated away from the zone along $\langle 011\rangle$ and $\langle 111\rangle$ directions as focused replacement sequences. The measured distances of these SIA from the zone along $\langle 011\rangle$ and $\langle 111\rangle$ directions were between $45 \AA$ to $150 \AA$, and $45 \AA$ to $85 \AA$ respectively. Unfortunately, the upper and lower limits of these distances were determined by a geometric sampling problem and not by the intrinsic range of focused replacement sequences (Beavan et al 1971).

We note that the features of the depleted zone reported in the previous section are in general qualitative agreement with many of the details of Beeler's (1966) computer simulated model. In particular, the elongation along $\langle 011\rangle$ directions arose in Beeler's model because of quasichannelling events. The 160 displaced atomic sites which were detected are fewer than the 200 predicted by the Kinchin and Pease (1955) model, but are greater than the average of 106 displaced atoms per depleted zone calculated by Beeler. The fact that the number observed is less than the number predicted by the Kinchin-Pease model is most likely due to the recombination of vacancies and SIA which originated from different displacement events within the cascade. It is clear that the depleted zone described here may not be characteristic of the average zone produced by a $20 \mathrm{keV}$ primary, and that it would be possible and desirable, to analyse a number of zones in order to obtain a better average representation.

\subsection{General remarks}

The detailed information reported concerning the defect structure of a depleted zone could not have been obtained if the field evaporation increment was as coarse as one atomic layer. The other applications of the FIM to the study of point defect clusters in irradiated metals have been performed by Attardo and Galligan (1966b), Hudson et al (1970), Buswell (1970) and Hudson and Ralph (1972). These FIM investigations have been of an essentially qualitative nature as they have given little quantitative information about the defect structure of depleted zones. The main difficulty with these other investigations is that fine enough field evaporation dissection increments were not employed. We believe that it is necessary to dissect a given atomic plane one or two atoms at a time, whereas all of the above cited experiments employed a minimum field evaporation increment of one atomic layer or greater.

\section{Vacancy defects in quenched platinum}

\subsection{Physical principles of the experiment}

At the thermal equilibrium the vacancy defect population can be expressed in the general form

$$
\begin{aligned}
c_{t}= & c_{1 \mathrm{v}} \\
= & \alpha_{1} \exp \left(S_{1 \mathrm{v}}^{\mathrm{f}} / k\right) \exp \left(-E_{1 \mathrm{v}}^{\mathrm{f}} / k T\right)+2 \alpha_{2} \exp \left(S_{2 \mathrm{v}}^{\mathrm{f}} / k\right) \exp \left(-E_{2 \mathrm{v}}^{\mathrm{f}} / k T\right) \\
& \quad+\ldots+n \alpha_{n} \exp \left(S_{n v}^{\mathrm{f}}\right) \exp \left(-E_{n \mathrm{v}}^{\mathrm{f}} / k T\right)
\end{aligned}
$$

where $n$ indicates the size of the vacancy cluster, $\alpha_{n}$ is a constant related to the lattice geometry of the cluster, $S_{n v}^{\mathrm{f}}$ and $E_{n \mathrm{v}}^{\mathrm{f}}$ are the entropy and energy of formation of a cluster of size $n$ (these quantities may be temperature dependent (eg see Seeger and Mehrer 
(1970)). When a specimen is quenched rapidly from a temperature $T_{\mathrm{q}}$ the defects become supersaturated and tend to cluster to an extent which is governed by the different positive binding energies of the clusters. For the purpose of the experiments performed we need only consider an assembly consisting of monovacancies and divacanciest. For this assembly equation (7) becomes

$$
c_{\mathrm{t}}=c_{1 \mathrm{v}}+2 c_{2 \mathrm{v}}
$$

where

$$
\begin{aligned}
& c_{1 \mathrm{v}}=\exp \left(S_{1 \mathrm{v}}^{\mathrm{f}} / k\right) \exp \left(-E_{1 \mathrm{v}}^{\mathrm{f}} / k T\right) \\
& c_{2 \mathrm{v}}=6\left(c_{1 \mathrm{v}}\right)^{2} \exp \left(-S_{2 \mathrm{v}}^{\mathrm{b}} / k\right) \exp \left(E_{2 \mathrm{v}}^{\mathrm{b}} / k T\right) \\
& E_{2 \mathrm{v}}^{\mathrm{b}}=2 E_{1 \mathrm{v}}^{\mathrm{f}}-E_{2 \mathrm{v}}^{\mathrm{f}} \\
& S_{2 \mathrm{v}}^{\mathrm{b}}=2 S_{1 \mathrm{v}}^{\mathrm{f}}-S_{2 \mathrm{v}}^{\mathrm{f}} .
\end{aligned}
$$

The quantities $E_{2 v}^{b}$ and $S_{2 v}^{b}$ are the divacancy binding energy and binding entropy respectively. If the specimen is quenched rapidly enough so that defect losses are small compared to the initial value of $c_{\mathrm{t}}$ at $T_{\mathrm{q}}$ then we can ignore these losses and consider only the redistribution of $c_{1 v}$ and $c_{2 v}$ during the quench. For a significant portion of the quench $c_{1 v}$ and $c_{2 v}$ are able to maintain quasiequilibrium conditions so that the populations of each defect follow equation (8). At a critical temperature $\left(T^{*}\right)$ the diffusivities of the defects become so small that the necessary association and dissociation rates are not large enough for the assembly to maintain itself in a quasiequilibrium state. Further quenching of the system does not change the concentrations characteristic of $T^{*}$ and the relative population of defects at $T^{*}$ then becomes 'frozen in'. The final 'frozen in' value of $c_{2 \mathrm{v}} / c_{1 \mathrm{v}}$ is very sensitive to the value of the binding free energy, $G_{2 \mathrm{v}}^{\mathrm{b}}=E_{2 \mathrm{v}}^{\mathrm{b}}-T S_{2 \mathrm{v}}^{\mathrm{b}}$ and $T_{\mathrm{q}}$, and relatively insensitive to the quenching rate (Balluffi et al 1970). Thus, the direct measurement of $c_{2 \mathrm{v}} / c_{1 \mathrm{v}}$ in a specimen which is quenched under carefully controlled conditions provides a measure of $G_{2 \mathrm{v}}^{\mathrm{b}}$.

\subsection{General procedure and results on control and quenched specimens}

High purity platinum $\left(\mathscr{R}=(3 \pm 1) \times 10^{3}\right)$ wire specimens were brine quenched from $1700 \pm 1^{\circ} \mathrm{C}$, and the $4 \cdot 2 \mathrm{~K}$ resistivity of the quenched in vacancy defects was measured. FIM specimens were then prepared from these same wires and the vacancy defects were imaged directly in an FIM as each specimen was dissected by pulse field evaporation at the rate of about 35 pulses per atomic layer (Berger et al 1972). A total of about $32000 \mathrm{ft}$ of film were scanned for defects. A total of 157 monovacancies and 9 divacancies were found in 593800 sites in three as quenched specimens and 76 monovacancies were found in 321000 sites in five specimens which were partially annealed after quenching.

In order to correct for the presence of possible surface artifact vacancy defects, 319600 sites were scanned in well annealed specimens under identical conditions and only 11 artifact monovacancies were found in the $\{135\},\{023\},\{124\},\{123\}$ and $\{167\}$ planes used for the present study (see table 1). It is also seen from table 1 that abnormally high artifact vacancy concentrations were detected in the $\{012\}$ and $\{137\}$ planes. Additional control experiments on platinum (Pétroff and Seidman 1972b) are in agreement with the findings presented in table 1. Furthermore, Speicher et al (1966) measured a concentration of $7.5 \times 10^{-3}$ at fr artifact vacancies in the $\{210\}$ plane of a well annealed platinum

+ The term divacancy shall refer to a pair of single vacant sites which are nearest neighbours of each other. 

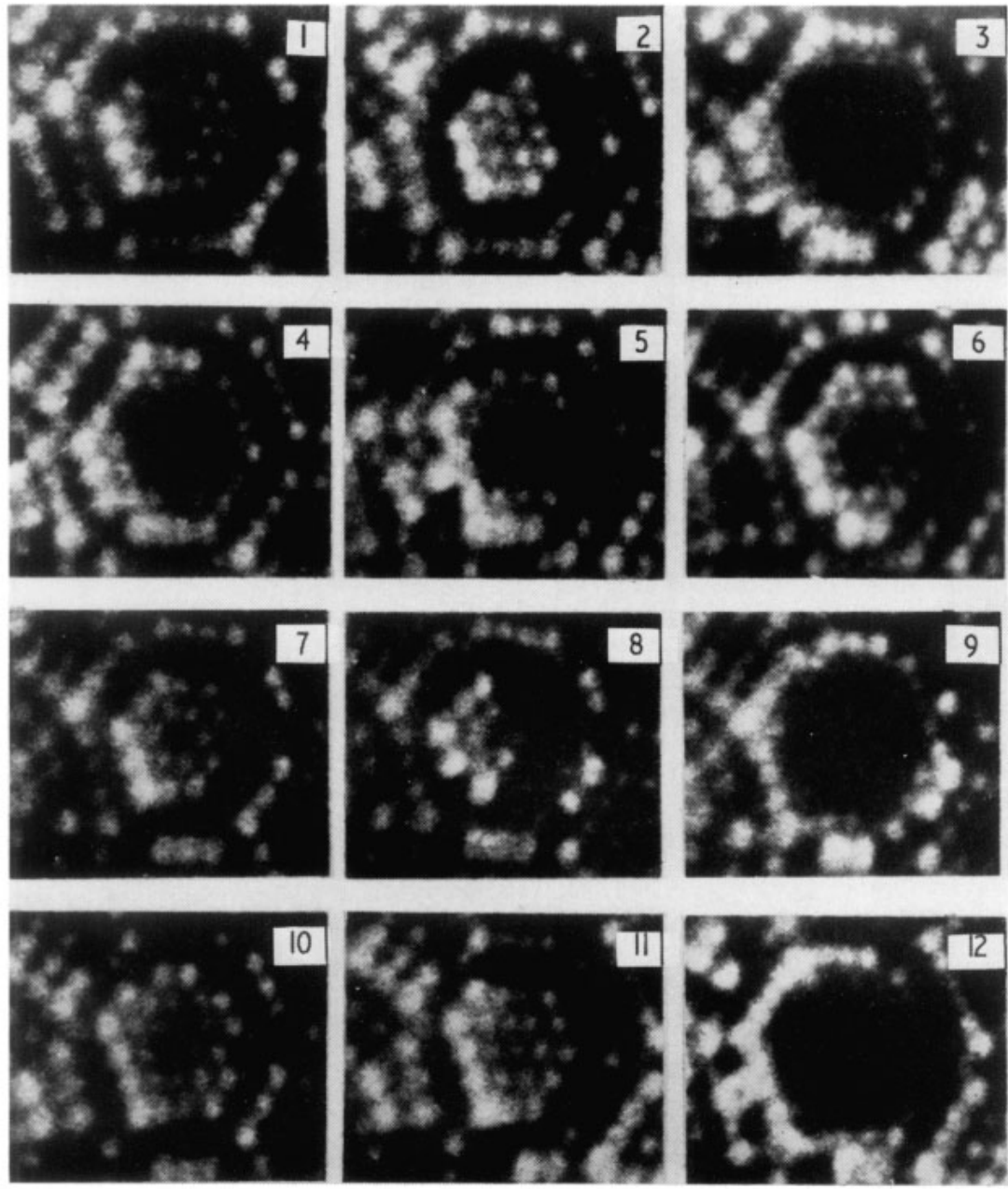

Figure 15. A monovacancy in a quenched platinum specimen revealed by the pulse dissection of successive (315) planes. The dissection of a plane directly above the monovacancy, the plane containing the monovacancy, and the plane directly below the monovacancy is shown in frames 1 to 3,4 to 9 , and 10 to 12 , respectively. 


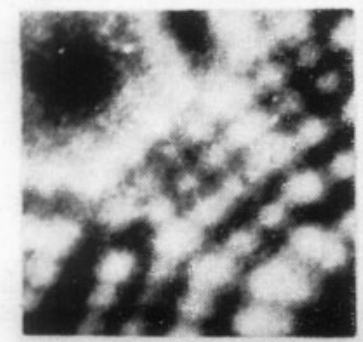

I
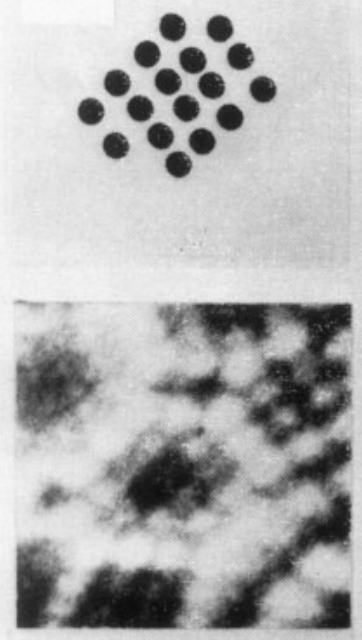

4

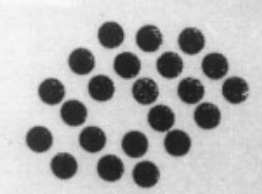

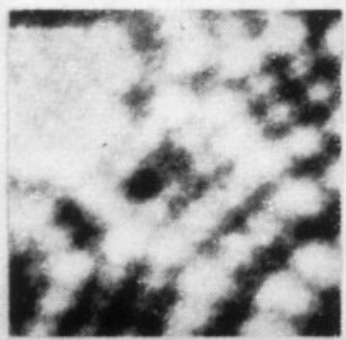

2
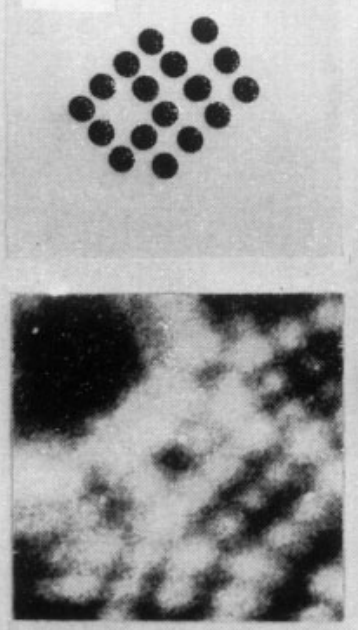

5

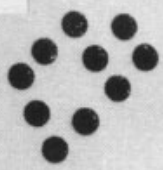

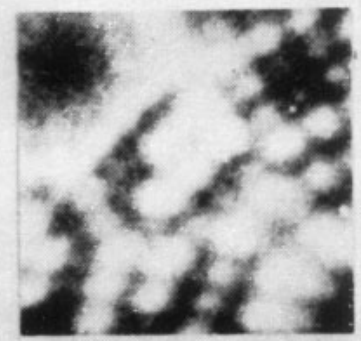

3
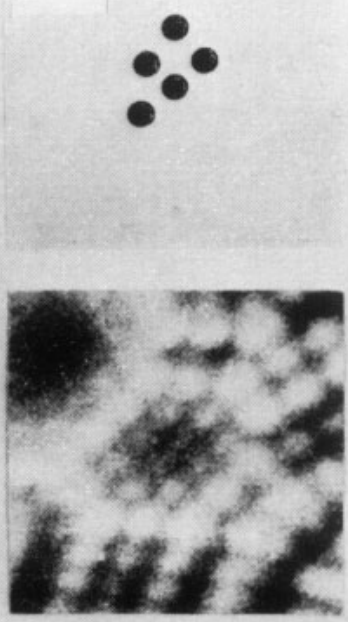

6

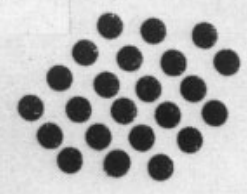

Figure 16. A divacancy in a quenched platinum specimen revealed by the pulse dissection of four successive (315) planes. The divacancy straddled two successive (315) planes. Frame 1 shows the perfect (315) plane immediately above the divacancy, frames 2 and 3 show the dissection of the plane containing the first member of the divacancy, frames 4 and 5 show the dissection of the next plane containing the second member of the divacancy, and frame 6 shows that perfect plane underneath the divacancy, The two vacant sites in the two successive (315) planes were shown to be connected by an $\frac{1}{2} a_{0}$ [110] vector, hence they are first nearest neighbours. 
specimen. Thus we may conclude that Müller's (1959) early experiment on quenched platinum, where monovacancies were detected in the $\{210\}$ planes, has no bearing on the quantitative problem of thermally generated vacancies.

Table 1. Artifact surface vacancy concentrations found on various planes of well annealed control specimens of platinum

\begin{tabular}{|c|c|c|c|c|}
\hline Plane & $\begin{array}{l}\text { Number of } \\
\text { atoms } \\
\text { counted }\end{array}$ & $\begin{array}{l}\text { Number of } \\
\text { artifact } \\
\text { vacant sites } \\
\text { counted }\end{array}$ & $\begin{array}{l}\text { Artifact } \\
\text { monovacancy } \\
\text { concentration }\end{array}$ & Remarks \\
\hline$\{012\}$ & 29360 & 420 & $1.43 \times 10^{-2}$ & \multirow{2}{*}{$\begin{array}{l}\text { Abnormally high artifact } \\
\text { monovacancy } \\
\text { concentrations }\end{array}$} \\
\hline$\{137\}$ & 23700 & 28 & $1.18 \times 10^{-3}$ & \\
\hline$\{135\}$ & 58360 & 2 & $3.42 \times 10^{-5}$ & \multirow{6}{*}{$\begin{array}{l}\text { Level of artifact } \\
\text { monovacancy } \\
\text { concentrations generated } \\
\text { acceptable for quenched in } \\
\text { vacancy defect counting }\end{array}$} \\
\hline$\{023\}$ & 42521 & 3 & $7 \cdot 14 \times 10^{-5}$ & \\
\hline$\{124\}$ & 103241 & 2 & $1.94 \times 10^{-6}$ & \\
\hline$\{123\}$ & 33512 & 1 & $2.99 \times 10^{-5}$ & \\
\hline$\{167\}$ & 81918 & 3 & $3.71 \times 10^{-5}$ & \\
\hline Total & $319552^{\mathrm{a}}$ & $11^{\mathrm{a}}$ & $3.45 \times 10^{-5 b}$ & \\
\hline
\end{tabular}

a This result does not include atoms or vacant sites from the $\{012\}$ and $\{137\}$ planes.

b This result is an average value excluding data for the $\{012\}$ and $\{137\}$ planes.

8.2.1. Quenched specimens. A pulse dissection sequence of a (315) plane containing a monovacancy in a quenched specimen is shown in figure 15 (plate). Frames 4 to 9 show the plane which contained the monovacancy. These five frames are representative of the $\sim 50$ frames of film which were recorded between frames 4 and 9 . Figure 16 (plate) is an example of a pulse dissection sequence through a divacancy which straddled two successive (315) planes. The first member of the divacancy located in the first (315) plane is seen in frame 2 and the second member located in the next (315) plane immediately below is seen in frames 4 and 5 . These two vacant sites were shown to be connected by an $\frac{1}{2} a_{0}[110]$ lattice vector by plotting the atom positions and vacant sites and comparing these maps with the known crystallography of the first (315) plane (Nicholas 1965).

The results for the five specimens which were polished using electropolishing procedure A (an initial polish at $-35^{\circ} \mathrm{C}$ followed by a polish in the temperature range 325 to $369^{\circ} \mathrm{C}$ for about $25 \mathrm{~s}$ ) are shown in figure $17(a)$. The quantity $c_{t}^{*}$ for each $\{h k l\}$ plane is corrected for the background artifact monovacancy concentration previously measured (table 1) in that plane. The weighted mean value of $c_{1}^{*}$ values for all $\{h k l\}$ planes was $\overline{c_{t}^{*}}=(2.15 \pm 0.59) \times 10^{-4}$ at fr†. In order to calculate this quantity, the $c_{t}^{*}$ value for each plane was weighted with respect to the value of the total number of atoms for that plane. It is seen that the individual values of $c_{t}^{*}$ all lie within approximately the standard deviation of the mean of $\overline{c_{t}^{*}}$ with the exception of the value for the $\{023\}$ planes.

+ The indicated uncertainty is equal to the standard deviation of the mean $\left(\sigma_{\mathrm{m}}\right)$ where $\sigma_{\mathrm{m}}=\sigma / \sqrt{l}$ (eg see Parratt 1961) and $\sigma=\left\{(1 / l) \Sigma_{i=1}^{i}\left|d_{i}\right|^{2}\right\}^{1 / 2}$ where the deviation $d_{i}$ is equal to $c_{\mathrm{t}}^{*}-\overline{c_{t}^{*}}$ and $l$ is the number of observations. The same definition was employed to calculate $\sigma_{\mathrm{m}}$ for $\rho_{1 \mathrm{v}} c_{1 \mathrm{v}}^{*}$, and $c_{2 \mathrm{v}}^{*}$. 
The weighted mean value of the monovacancy resistivity for all specimens $\left(\bar{\rho}_{1 v}\right)$ for the specimens electropolished by procedure A was $(4.8 \pm 1.4) \times 10^{-4} \Omega \mathrm{cm}(\mathrm{at} \mathrm{fr})^{-1}$. To calculate $\rho_{1 \mathrm{v}}$ it was assumed that the resistivity of a divacancy was twice that of two isolated monovacancies (Bross and Seeger 1958, Flynn 1962). The values of $\rho_{1 \mathrm{v}}$ for each specimen were calculated after correcting the concentration data for each specimen for an average background artifact monovacancy concentration of $3.4 \times 10^{-5}$ at $\mathrm{fr}$ (table 1 ). The quantity $\bar{\rho}_{1 \mathrm{v}}$ was then calculated by weighting the value of $\rho_{1 \mathrm{v}}$ for each specimen by the total number of atoms counted for that specimen.

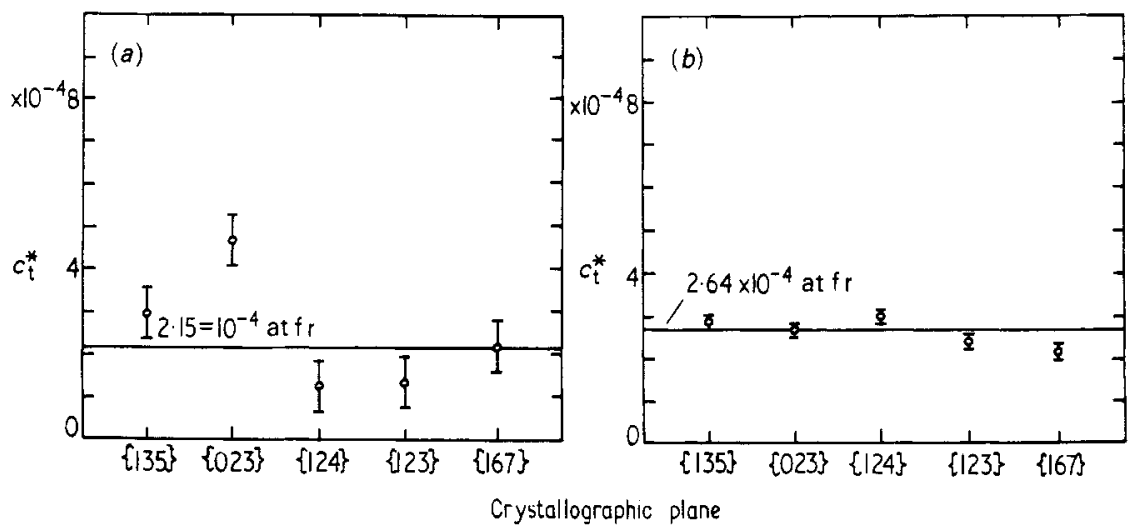

Figure 17. (a) A plot of $c_{t}^{*}$ against the $\{h k l\}$ plane in which this quantity was measured. These specimens were given a brief anneal in a molten salt solution prior to their examination in the FIM. The weighted mean concentration $\overline{\left(c_{t}^{*}\right)}$ is indicated by the horizontal line and the error bar on each point represents \pm 1 standard deviation of the mean $\left(\sigma_{\mathrm{m}}\right)$. Number of vacant sites, 78: atomic sites, 321 298. (b) A plot of $c_{1}^{*}$ against $\{h k l\}$ plane in which this quantity was measured. These specimens were polished totally at room temperature. The quantity $\overline{c_{t}^{*}}$ is represented by the horizontal line and the error bar on each point is equal to \pm 1 standard deviation of the mean $\left(\sigma_{\mathrm{m}}\right)$ ( $\mathrm{see} \$ 8.2 .1$ for the definition of $\sigma_{\mathrm{m}}$ ). Number of vacant sites, 175: atomic sites, 593794.

The results for the specimens which were polished only at room temperature (procedure B) are shown in figure $17(b)$. The value of $\overline{c_{t}^{*}}$ is $(2.64 \pm 0.14) \times 10^{-4}$ and this value is compared to $c_{1}^{*}$ for each $\{h k l\}$ plane in this figure. It is seen that all of the values of $c_{t}^{*}$ lie within one to two $\sigma_{\mathrm{m}}$ of $\overline{c_{\mathrm{t}}^{*}}$ with the exception of $c_{\mathrm{t}}^{*}$ for the $\{167\}$ plane.

Nine divacancies were detected in the 593800 atomic sites in this set of specimens which were not heated above room temperature during electropolishing. This result is to be compared to the previous results where only one divacancy was found in 321300 sites in the group of specimens which were given a brief high temperature electropolish. The weighted mean divacancy concentration $\left(\overline{c_{2}^{*}}\right)$ as $(1.68 \pm 0.48) \times 10^{-5}$ at fr, hence, the ratio $\left(\overline{c_{2 v}^{*}} / c_{1 v}^{*}\right)$ was $0.06 \pm 0.02$. This ratio may be used to estimate $G_{2 \mathrm{v}}^{\mathrm{b}}$ (see $\$ 8.4$.).

The value of $\overline{\rho_{1 \mathrm{v}}}$ for the specimens electropolished by procedure $\mathrm{B}$ was $(5.75 \pm 0.30)$ $\times 10^{-4} \Omega \mathrm{cm}$ (at fr) ${ }^{-1}$ which is in agreement with the value obtained for the specimens electropolished by procedure A. 


\subsection{Discussion of experimental results}

The first result which our experiments demonstrated is that vacant lattice sites are the dominant defects in thermal equilibrium at elevated temperatures in platinum. This was proven by the direct observation of the quenched in vacancy defect population via the FIM. This is a significant result because of the absence of any combined length and lattice parameter change measurements (Simmons and Balluffi 1960,1962,1963) on platinum at elevated temperature. Furthermore, it was determined that the defect population consisted mainly of monovacancies and divacancies and that the monovacancy was the preponderant defect at thermal equilibrium and after rapid quenching. The ratio of the divacancy and monovacancy concentrations after quenching from $1700^{\circ} \mathrm{C}$ to room temperature was $\overline{c_{2 \mathrm{v}}^{*}} \overline{c_{1 \mathrm{v}}^{*}}=0.06 \pm 0.02$.

The second result obtained was the total concentration of vacant sites after quenching from $1700 \pm 1^{\circ} \mathrm{C}$. The values of $\overline{c_{t}^{*}}$ were $(2.15 \pm 0.59) \times 10^{-4}$ and $(2.64 \pm 0.14) \times 10^{-4}$ at fr for the two groups of specimens subjected to differing electropolishing treatments. Both of these values must be lower than the thermal equilibrium value at $1700^{\circ} \mathrm{C}$ due to the vacancy losses to sinks which occurs in any non ideal quench (eg see Seidman and Balluffi 1966, 1967 and Balluffi et al 1970). In addition $\overline{c_{1}^{*}}$ for the first group of specimens (electropolishing procedure A) is expected to be slightly lower than for the second group (electropolishing procedure B), since the former specimens were subjected to a high temperature electropolish which resulted in a loss of the relatively small divacancy population to the specimen surfaces.

The third important result emerged as a direct consequence of the different electropolishing treatments employed. For the specimens which were given a finishing high temperature electropolish in a molten salt solution $\overline{c_{2 \mathrm{v}}^{*}}$ was a factor of less than or about five lower than for the specimens which were polished totally at room temperature. This result indicated that the divacancies were mobile enough at the elevated polishing temperature to migrate out of the FIM tips, whereas the mean monovacancy concentrations differed by only about $16 \%$ with the specimens polished by procedure $\mathrm{A}$ having the lower monovacancy concentration. Thus we are led to the conclusion that the divacancy in platinum must be more mobile than the monovacancy. The same conclusion was reached by Pétroff and Seidman (1972b) in a study of the temperature dependence (from 77 to $508 \mathrm{~K}$ ) of $c_{1 v}$ and $s_{2 \mathrm{v}}$ produced in platinum specimens by irradiation with 20 or $30 \mathrm{keV} \mathrm{Pt}{ }^{+}$ ions at 10 or $11 \mathrm{~K}$. The fact that a divacancy should be more mobile than a monovacancy was first suggested by Seitz (1952), then elaborated on in a number of subsequent theoretical calculations (eg Bartlett and Dienes 1953 and Damask et al 1959) and used numerous times in the interpretation of the recovery behaviour of quenched metals (eg Bauerle and Koehler 1957 and Koehler et al 1957). The present observations, along with the Pétroff and Seidman (1972b) results, constitute the first direct pieces of evidence regarding the relative mobilities of these defects in any metal.

Finally, the fourth result was an absolute upper limit to the value for $\bar{\rho}_{1 v}$. It is emphasized that the quenched in resistivity increment and $\overline{c_{t}^{*}}$ were measured on the same sets of specimens, hence the values of $\bar{\rho}_{1 \mathrm{v}}$ obtained represent the first direct measurements of this quantity for platinum. The reported values are upper limits to $\bar{\rho}_{1 \mathrm{v}}$, since the measured values of $\overline{c_{t}^{*}}$ are lower limits because of possible vacancy losses during the electropolishing operations. The preferred value for $\bar{\rho}_{1 \mathrm{v}}$ is $(5.75 \pm 0.30) \times 10^{-4} \Omega \mathrm{cm}(\mathrm{at} \mathrm{fr})^{-1}$, because the quenched in resistivity increment was a constant, electropolishing operations were done at room temperature to minimize vacancy losses and the value of $\overline{c_{t}^{*}}$ showed the least scatter for the specimens electropolished by procedure $\mathbf{B}$. 


\subsection{The divacancy binding free energy}

A detailed series of calculations were first performed (Seidman et al 1972) which showed that the measured ratio of divacancy concentration to monovacancy concentration $\left(c_{2 \mathrm{v}} / c_{1 \mathrm{v}}\right)$ for the specimens electropolished by procedure $\mathrm{B}$ was not affected by specimen storage and electropolishing procedures subsequent to the quench and hence represented $c_{2 \mathrm{v}} / c_{1 \mathrm{v}}$ at $T^{*}$.

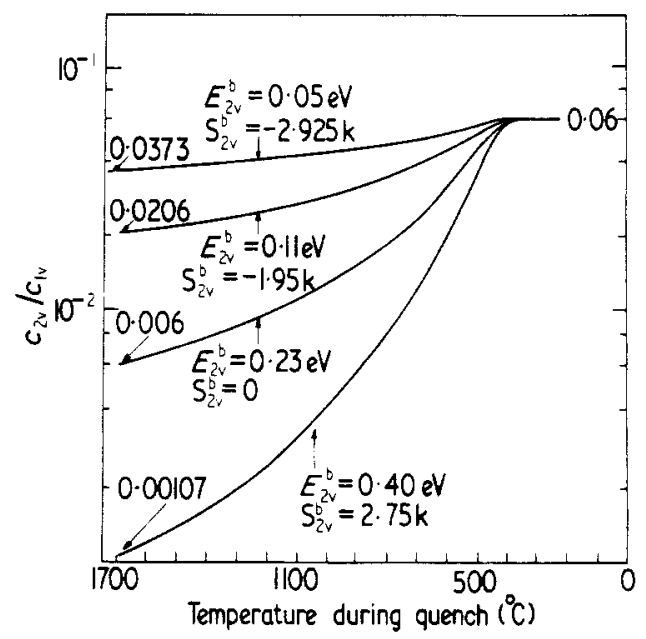

Figure 18. The ratio of the divacancy to monovacancy concentration $\left(c_{2 v} / c_{1 v}\right)$ against temperature during a quench from $1700^{\circ} \mathrm{C}$ for different combinations of $E_{2 \mathrm{v}}^{\mathrm{b}}$ and $S_{2 \mathrm{v}}^{\mathrm{b}}$ which lead to the same value of $c_{2 v} / c_{1 v}$ at room temperature. The curves were calculated for a closed system of monovacancies and divacancies in platinum with a total concentration of $2.64 \times 10^{-4}$ at $\mathrm{fr}, E_{1 \mathrm{v}}^{\mathrm{m}}=1.38 \mathrm{eV}, v=10^{13} \mathrm{~s}^{-1}$ and an experimental quenching rate expression.

Next the value of $c_{2 v} / c_{1 v}$ at $T^{*}$ for a quench from $1700^{\circ} \mathrm{C}$ was calculated by solving numerically the coupled nonlinear differential equations governing $c_{2 \mathrm{v}}$ and $c_{1 \mathrm{v}}$ (eg Damask and Dienes 1962) during a quench. In calculating $c_{2 \mathrm{v}} / c_{1 \mathrm{v}}$ the experimentally determined quenching curve (Berger et al 1972) was used and $c_{\mathrm{t}}$ was set equal to $\overline{c_{\mathrm{t}}^{*}}$. The variable parameters used in this model were the monovacancy motion energy $E_{1 v}^{\mathrm{m}}$, a frequency factor $v$ and $G_{2 \mathrm{v}}^{\mathrm{b}}$. Calculations showed that the quenched in value of $c_{2 v} / c_{1 v}$ was a weak function of $E_{1 \mathrm{v}}^{\mathrm{m}}$ and $v$ but a very strong function of $G_{2 \mathrm{v}}^{\mathrm{b}}$. Finally, a search was made for all possible combinations of $E_{2 v}^{b}$ and $S_{2 v}^{b}$ which gave a quenched in value of $c_{2 \mathrm{v}} / c_{1 \mathrm{v}}=0.06 \pm 0.02$ for the parameters $\overline{c_{\mathrm{t}}^{*}}=2.64 \times 10^{-4}$ at fr, $E_{1 \mathrm{v}}^{\mathrm{m}}=1.38 \mathrm{eV}$ (Jackson 1965 and Schumacher et al 1968) and $v=10^{13} \mathrm{~s}^{-1}$. Typical results are shown in figure 18 , for $E_{2 \mathrm{v}}^{\mathrm{b}}=0.40,0.23,0.11$ and $0.05 \mathrm{eV}$. These calculations showed that the following linear relationship (in eV) exists between $E_{2 \mathrm{v}}^{\mathrm{b}}$ and $S_{2 \mathrm{v}}^{\mathrm{b}}$;

$$
E_{2 \mathrm{v}}^{\mathrm{b}}=\left\{(0.23 \pm 0.03)+6.17 \times 10^{-2}\left(S_{2 \mathrm{v}}^{\mathrm{b}} / k\right)\right\} .
$$

Equation (9) implies that for all combinations of $E_{2 v}^{b}$ and $S_{2 v}^{b}$ which lead to a fixed $c_{2 \mathrm{v}} / c_{1 \mathrm{v}}$ for a quench from one $T_{\mathrm{q}}$ that $G_{2 \mathrm{v}}^{\mathrm{b}}$ is a constant which is characteristic of a single $T^{*}$. This can be seen by noting that under the above conditions

$$
G_{2 \mathrm{v}}^{\mathrm{b}}\left(T^{*}\right)=E_{2 \mathrm{v}}^{\mathrm{b}}\left(T^{*}\right)-T^{*} S_{2 \mathrm{v}}^{\mathrm{b}}\left(T^{*}\right)=\text { constant. }
$$


Hence, a calculation showed that $T^{*}=443^{\circ} \mathrm{C}$ and $\mathrm{G}_{2 \mathrm{v}}^{\mathrm{b}}\left(443^{\circ} \mathrm{C}\right)=0.23 \mathrm{eV}$ for our experiments. We note that a further decomposition of $G_{2 v}^{b}$ and $E_{2 v}^{b}$ into $S_{2 v}^{b}$ requires a knowledge of a second value of $G_{2 v}^{\mathrm{b}}$ for a different $T^{*}$. This could be accomplished experimentally by quenching from the same $T_{\mathrm{q}}$ at a faster quenching rate than the one employed, or alternatively quenching from a different $T_{\mathrm{q}}$.

8.5. A re-examination of the platinum tracer diffusion data in terms of a monovacancy and divacancy model

In an attempt to decompose $G_{2 v}^{b}$ into $E_{2 v}^{b}$ and $S_{2 v}^{b}$ terms the available platinum tracer diffusion data (Kidson and Ross 1957 and Cattaneo et al 1962) were re-examined critically (Seidman et al 1972). It was concluded that the existing data for the tracer diffusion coefficient in platinum are not sufficiently accurate to allow a significant decomposition of $G_{2 \mathrm{v}}^{\mathrm{b}}$.

\section{Conclusions}

In this paper we have presented the results of FIM investigations of the fundamental properties of point defects in metals. The point defects were induced by either irradiation with heavy metal ions or rapid quenching from elevated temperatures. These experiments yielded direct results about the properties of the point defects that are not obtainable presently by other techniques. It is believed that these experiments and results have helped to establish the FIM as a quantitative instrument for the study of point defects in metals.

\section{Acknowledgments}

I would like to thank Professor R W Balluffi for much encouragement and useful discussion over the years. Thanks are also due to my past and present co-workers Drs Arnold S Berger, Kyoon H Lie, Pierre Pétroff and Mr Kenneth L Wilson for permission to quote unpublished research. In addition I want to thank Mr B F Addis for his help in the preparation of high purity single metal crystals, Mrs Karen Pratt for her aid in scanning film, and Mr Robert Whitmarsh for his enthusiastic technical assitance. The John Simon Guggenheim Memorial Foundation is also thanked for support funds during the preparation of this article.

\section{References}

Ast D G $1972 P h D$ Thesis Cornell University, Ithaca, New York

Ast D G and Seidman D N 1968 Appl. Phys. Lett. $13348-50$

_- 1970 Cornell University Mater. Sci. Center Rep. No 1322 1971 Surface Sci. 28 19-31

Attardo M J and Galligan J M 1966a Phys, Rev. Lett. 17 1173-5

1966b Phys. Rev. Lett. 17 191-3

Attardo M J, Galligan J M and Chow J G Y 1967 Phys. Rev. Lett. $1973-4$

Balluffi R W, Lie K H, Seidman D N and Siegel R W 1970 Vacancies and Interstitials in Metals eds A Seeger,

D Schumacher, W Schilling and J Diehl (Amsterdam: North Holland) pp 125-67

Bartlett J M and Dienes G J 1953 Phys. Rev. $89848-50$

Bauerle J E and Koehler J S 1957 Phys. Rev. 107 1493-8

Beavan L A, Scanlan R M and Seidman D N 1971 Acta Metall. 19 1339-50 
Beeler J R 1966 Phys. Rev. 150 470-87

Berger A S, Seidman D N and Balluffi R W 1972 Acta Metall. to be published

Bowkett K M and Smith D A 1970 Field-Ion Microscopy (Amsterdam: North Holland)

Brandon D G 1968 Field Ion Microscopy eds J J Hren and S Ranganathan (New York: Plenum) p 47

Bross H and Seeger A 1958 J. Phys. Chem. Solids 4 161-76

Burger G et al 1966 Phys. Lett. $20470-1$

Buswell J T 1970 Phil. Mag. 22 787-802

- 1971 Phil. Mag. 23 293-302

Cattaneo F, Germagnoli E and Grasso F 1962 Phil. Mag. 7 1373-83

Coltman R R, Klabunde C E and Redman J K 1967 Phys. Rev, 156 715-24

Corbett J W 1966 Solid State Physics, Suppl. 7 eds F Seitz and D Turnbull (New York: Academic Press) pp 200-01

_- 1970 Vacancies and Interstitials in Metals eds A Seeger, D Schumacher, W Schilling and J Diehl (Amsterdam: North Holland) pp 977-88

Corbett J W, Smith R B and Walker R M 1959a Phys. Rev. 114 1452-9 1959b Phys. Rev. $1141460-72$

Damask A C and Dienes G J 1962 Point Defects in Metals (New York: Gordon and Breach) pp 911-60

Damask A C, Dienes G J and Weizer V G 1959 Phys. Rev. $113781-4$

Dibbert H J, Sonnenberg K, Schilling W and Dedek U 1972 Jülich KFA Rep. No 859

DiCarlo J A, Snead C L and Goland A N 1969 Phys. Rev. 178 1059-72

Doyama M, Koeler J S, Lwin Y N, Ryan E A and Shaw D G 1971 Phys. Ret. B 4 281-91

Duesing G and Schilling W 1969 Rad. Effects 1 65-7

Erents $\mathrm{K}$ and Carter G 1966 Vacuum 16 523-7

Erents K, Layson R P W and Carter G $1967 \mathrm{~J}$. Vac. Sci. Tech. $4252-6$

Flynn C P 1962 J. Phys. Radium 23 654-8

Frank W 1971 Rad. Effects 8 145-54

Frank W and Seeger A 1969 Rad. Effects 1 117-31

Gregov B and Lawson R P W 1972 Can. J. Phys. 50 791-7

Hren J J and Ranganathan S 1968 Field Ion Microscopy (New York: Plenum)

Hudson J A, Dury B L and Ralph B 1970 Phil. Mag. $21779-85$

Hudson J A and Ralph B 1972 Phil. Mag. 25 265-80

Jackson J J 1965 Lattice Defects in Quenched Metals eds R M J Cotterill, M Doyama, J J Jackson and M Meshii (New York: Academic Press) pp 467-79

Johnson H H 1970 Scripta Metall. $4771-6$

Johnson R A 1964a Phys. Rev. 134 A1329-36

1964b Diffusion in Body Centered Cubic Metals (Metals Park, Ohio: American Society for Metals) p 357 1966 Phys. Rev. 145 423-33

Kidson C V and Ross R 1957 Proc. Int. Conf. Radioisotopes in Sci. Research vol 1 ed R C Extermann (London: Pergamon) pp 185-93

Kinchin G H and Pease R S 1955 Rep. Prog. Phys. 18 1-51

Koehler J S 1970 Vacancies and Interstitials in Metals eds A. Seeger, D. Schumacher, W. Schilling and J. Diehl (Amsterdam: North Holland) pp 989-98

Koehler J S 1970 Vacancies and Interstitials in Metals eds A Seeger, D Schumacher, W Schilling and J Diehl

Kornelsen E V 1972 Rad. Effects $13227-36$

Kunz W, Faber K, Lachenmann R and Schultz H 1972 Proc. Discussion Meeting Defects in Refractory Metals, Belgium 1971 ed R DeBatist at press

Moore A J W 1962 J. Phys. Chem. Solids $23907-12$ 1968 Field Ion Microscopy eds J J Hren and S Ranganathan (New York: Plenum) pp 69-87

Moore A J W and Ranganathan S 1967 Phil. Mag. 16 723-37

Müller E W 1959 Z. Phys. 156 399-410

Müller E W and Tsong T T 1969 Field Ion Microscopy (New York: Elsevier) p 72

Neeley H H, Keefer D W and Sosin A 1968 Phys. Stat. Solidi 32 675-82

Nicholas J F 1965 An Atlas of Models of Crystal Surfaces (New York: Gordon and Breach) pp 56-7

Nihoul J 1970 Vacancies and Interstitials in Metals eds A Seeger, D Schumacher, W Schilling and J Diehl (Amsterdam: North Holland) pp 839-88

O'Conner G P and Ralph B 1972a Phil. Mag. $26113-28$

- 1972b Phil. Mag. 26 129-42

Parratt L G 1961 Probability and Experimental Errors in Science (New York: Wiley) pp 92-4

Pétroff $P$ and Seidman D N 1971 Appl. Phys, Lett. 18 518-20

- 1972 a Acta Metall. to be published 
Pétroff P and Seidman D N 1972 b Cornell University Mater. Sci. Center Rep.

Roberts C G 1968a PhD Thesis University of North Carolina, Chapel Hill

_- 1968b Bull. Am. Phys. Soc. 13255

Robertson S H and Seidman D N 1968 J. Phys. E: Sci. Instrum. 1 1244-5

Robinson J T, Wilson K L and Seidman D N 1972 Phil. Mag. submitted for publication

Scanlan R M, Styris D L, Seidman D N 1971a Phil. Mag. 23 1439-57 1971 b Phil. Mag. 23 1459-78

Scanlan R M, Styris D L, Seidman D N and Ast D G 1969 Cornell University Maler. Sci. Center Rep. No 1159

Schilling W, Burger G, Isebeck D and Wenzl H 1970 Vacancies and Interstitials in Metals eds A Seeger, D Schumacher, W Schilling and J Diehl (Amsterdam: North Holland) pp 255-361

Schüle W, Frank W and Seeger A 1971 Rad. Effects 10 123-7

Schultz H 1968/69 Mater. Sci. 3 189-219

Schumacher D, Seeger A and Härlin O 1968 Phys. Stat. Solidi 25 359-71

Seeger A 1962 Radiation Damage in Solids (International Atomic Energy Agency: Vienna) pp 101-27

_- 1970 Vacancies and Interstitials in Metals eds A Seeger, D Schumacher, W Schilling and J Diehl (Amsterdam: North Holland) pp 999-1014

Seeger A and Mehrer H 1970 Vacancies and Interstitials in Metals eds A Seeger, D Schumacher, W Schilling and J Diehl (Amsterdam: North Holland) pp 1-50

Seidman D N and Balluffi R W 1966 Phys. Stat. Solidi $17531-41$

_ 1967 Lattice Defects and Their Interactions ed R R Hasiguti (New York: Gordon and Breach) pp 911-60

Seidman D N, Berger A S and Balluffi R W 1972 Acta Metall to be published

Seidman D N and Lie K H 1972 Acta Metall. 20 1045-60

Seidman D N and Scanlan R M 1971 Phil. Mag. 23 1429-37

Seidman D N, Scanlan R M, Styris D L and Bohlen J W 1969 J. Phys. E: Sci. Instmim. 2 473-76

Seitz F 1952 Adv. Phys. $143-90$

Simmons R O and Balluffi R W 1960 Phys. Rev. $119600-5$

- 1962 Phys. Rev. $125862-72$

1963 Phys. Rev. 129 1533-44

Simpson H M and Sosin A 1970a Rad. Effects 2 299-302 1970b Rad. Effects 3 1-21

Sinha M K and Müller E W $1964 J$. appl. Phys. 35 1256-61

Smith P J and Smith D A 1970 Phil. Mag. 21 907-12

Sonnenberg K, Schilling W, Dibbert H J, Mika K and Schröder K 1971 Jülich KF A Rep.

Sosin A 1967 Lattice Defects and Their Interactions ed R R Hasiguti (New York: Gordon and Breach) pp 235-66

Sosin A and Bauer W 1966 Phys. Rev. 147 478-81

Speicher C A, Pimbley W T, Attardo M J, Galligan J M and Brenner S S 1966 Phys. Lett. $23194-6$

Takamura S, Hanada R, Okuda S and Kimura H 1971 J. Phys. Soc. Japan 30 1091-5

Venables J A 1970 Atomic Collision Phenomena in Solids eds D W Palmer, M W Thompson and P D Townsend (Amsterdam: North Holland) pp 132-61

Venables J A and Thomas G 1970 Vacancies and Interstitials in Metals eds A Seeger, D Schumacher, W Schilling and J Diehl (Amsterdam: North Holland) pp 531-55

Wollenberger H 1971 Rad. Effects 10 135-6 